\title{
CONSIDERACIONES DE USO DE LA ENERGÍA PLÁSTICA EN EL DISEÑO SÍSMICO
}

\author{
Amador Terán-Gilmore ${ }^{(1)}$
}

\section{RESUMEN}

El diseño sísmico racional de una estructura debe contemplar el control de su nivel de daño estructural cuando se le sujeta a la excitación sísmica de diseño, la cual debe estar constituida por una familia de movimientos del terreno. Estrictamente hablando, el control del daño estructural solo es posible cuando se hace una consideración explícita de las demandas de deformación plástica en la estructura, lo que implica no solo conocer su magnitud, sino su secuencia de ocurrencia a través del tiempo. La similitud de los niveles de daño estructural promedio, estimados por medio de dos índices de daño (uno que toma en cuenta la secuencia de ocurrencia de las demandas de deformación plástica y otro que ignora dicha secuencia), en sistemas de un grado de libertad sujetos a la acción de diferentes familias de movimientos del terreno, sugiere que es posible plantear el control del daño estructural en estructuras sismorresistentes a partir del uso de la energía plástica que disipa la estructura como representación de las demandas plásticas acumuladas. Dentro de este contexto, se observa además que las demandas máxima y acumuladas de deformación en edificios regulares, diseñados conforme a los requerimientos de los códigos actuales de diseño sísmico, son similares a aquellas obtenidas, para la misma familia de movimientos del terreno, a partir de espectros de respuesta. Lo anterior aporta las bases para plantear que el control de daño estructural en una estructura sismorresistente regular puede establecerse a partir de las demandas de deformación máxima y de energía plástica leídas directamente de espectros de respuesta.

\section{SUMMARY}

The rational seismic design of a structure must contemplate the control of its level of structural damage when it is subjected to the design seismic excitation, which is normally constituted by a family of ground motions. Strictly speaking, structural damage control is only possible through the explicit consideration of the plastic deformation demands in the structure, which requires the knowledge of their magnitude and sequence of occurrence. The similitude of the average levels of structural damage, estimated by using two damage indices (one that takes into account the sequence of occurrence of the plastic deformation demands and another one that neglects this sequence), in single degree of freedom systems subjected to different families of ground motion, suggests that damage control in an earthquake-resistant structure can be formulated through the use of the plastic energy dissipated by that structure in lieu of a more accurate representation of the cumulative plastic deformation demands. Within this context, it has been found that the maximum and cumulative deformation demands in regular buildings, designed according to the requirements of current seismic design codes, are similar to those obtained, for the same family of ground motions, from response spectra. The latter fact suggests that structural damage control in a regular earthquake-resistant structure can be formulated through the use of the maximum displacement and plastic energy demands read directly from response spectra.

Artículo recibido el 16 de enero de 2001 y aprobado para su publicación el 14 de agosto de 2001. Se aceptarán comentarios y/o discusiones hasta cinco meses después de su publicación.

(1) Departamento de Materiales, Universidad Autónoma Metropolitana Azcapotzalco, Edificio H, 3er Piso, Av. San Pablo \# 180, Col. Reynosa Tamaulipas, 02200 México, DF. e-mail: tga@correo.azc.uam.mx 


\section{INTRODUCCIÓN}

Actualmente varios investigadores e ingenieros prácticos promueven la actualización del diseño sísmico por medio de metodologías de diseño basadas en el control de las demandas máximas de deformación; en particular, de las demandas máximas de desplazamiento lateral. Esto puede ilustrarse a partir de las recomendaciones generales que se derivaron del Simposio Internacional de Metodologías de Diseño para la siguiente Generación de Códigos (Fajfar y Krawinkler, 1997), en particular de la siguiente: "Parece ser que el enfoque mejor adaptado para alcanzar los objetivos de un diseño sísmico por desempeño es un diseño de control de deformaciones”.

El enfoque actual de diseño sísmico para estructuras estándar acepta la posibilidad de que estas incurran, de manera importante, en su rango de comportamiento plástico durante excitaciones sísmicas severas. La evidencia experimental y de campo sugiere fuertemente que las características mecánicas de una estructura se deterioran, en algunas ocasiones considerablemente, cada vez que se introduce a esta de manera importante dentro de su rango de comportamiento plástico. Este fenómeno, denotado en este artículo como fatiga de bajo ciclaje, puede llegar a ser importante durante sismos de larga duración, durante los cuales las estructuras pueden estar sujetas a varias reversiones importantes de deformación plástica. Las características mecánicas de las estructuras exhiben un deterioro importante cuando no se controla el número y magnitud de los ciclos de comportamiento plástico, de tal manera que su desempeño sísmico puede llegar a alejarse decisivamente de aquel esperado para una estructura de ocupación estándar.

La insistencia de algunos investigadores (por ejemplo, véase Bertero, Bertero y TeranGilmore, 1996; y Bertero y Bertero, 2000) en la importancia de considerar las demandas acumuladas de deformación plástica llevaron al grupo de trabajo involucrado en el Simposio Internacional de Metodologías de Diseño para la siguiente Generación de Códigos a aclarar: "Deberá considerarse el daño acumulado (energía disipada) en el diseño sísmico de: estructuras con elementos que presentan deterioro rápido; sismos de larga duración. Deberá incluirse implícitamente la energía (o cualquier otra caracterización de los efectos de la duración) en la determinación de deformaciones objetivo que tomen en cuenta los efectos de la acumulación de daño en la capacidad de deformación última."

Para ilustrar lo planteado hasta ahora, considere los espectros de resistencia mostrados en la Figura 1a, y que corresponden a diferentes demandas máximas de ductilidad $(\mu)$ para la componente este-oeste del movimiento registrado durante 1985 en la Secretaría de Comunicaciones y Transportes (SCT EO). Esta excitación esta caracterizada por un periodo dominante ( $\left.T_{g}\right)$ de 2 seg y una banda angosta de frecuencias alrededor de este $T_{g}$. Las ordenadas del espectro de resistencia elástico $(\mu=1)$ exhiben una notoria variación con respecto al valor del periodo $(T)$. En particular, las demandas de resistencia aumentan de manera considerable para estructuras con $T$ cercano a $T_{g}$, lo que hace necesario tomar precauciones pertinentes si se decide diseñar una estructura para que permanezca elástica en este rango de $T$. Sin embargo, esta variación se reduce significativamente en los espectros inelásticos, de manera que para estructuras capaces de desarrollar comportamiento plástico caracterizado por $\mu \geq 2$, los espectros inelásticos sugieren que no hay nada de particular en las demandas de resistencia para $T$ cercano a $T_{g}$. Esta noción se ve reforzada por los espectros 
inelásticos de desplazamiento mostrados en la Figura 1 b, donde para $\mu \geq 2$ y $T$ cercano a $T_{g}$, el desplazamiento solo muestra ligeras variaciones con respecto a los valores de $T$ y $\mu$.

A pesar de lo sugerido por los espectros de resistencia y desplazamiento, existe una gran diferencia en el comportamiento de estructuras con diferente $T$ cuando se les sujeta a SCT EO, particularmente cuando este $T$ se acerca al valor de $T_{g}$. A manera de ilustración, considere espectros de energía plástica $\left(E_{H \mu}\right)$, definida como la energía que disipa la estructura en su rango plástico de comportamiento plástico durante la excitación sísmica. La Figura 1c muestra que, independientemente del valor de $\mu$, hay un incremento importante en la energía disipada conforme $T$ se aproxima a $T_{g}$. La Figura 1d, que muestra espectros de número de reversiones plásticas (NRP), definido como el número de veces que un sistema incurre en su rango plástico de comportamiento una vez que ha incurrido en el mismo en dirección contraria, completa al panorama al mostrar que el número de veces que una estructura incurre en su rango plástico de comportamiento crece significativamente conforme el valor de $T$ se aproxima al de $T_{g}$ y conforme el valor de $\mu$ se incrementa. Si un diseñador tuviera acceso a espectros como los mostrados en las Figuras 1c y 1d, podría decidir, con el fin de evitar problemas de fatiga de bajo ciclaje, no ubicar su estructura en un rango de $T$ cercano a $T_{g}$, o incluso diseñarla para valores pequeños de $\mu$; decisiones que no podrían plantearse a partir del uso de los espectros inelásticos de resistencia y desplazamiento mostrados en las Figuras 1a y 1b, respectivamente.

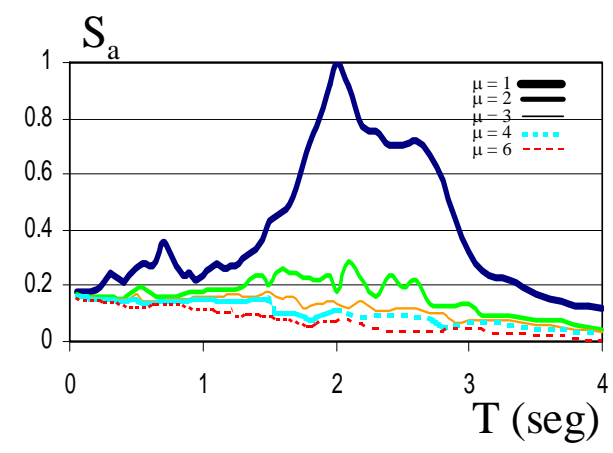

a) Resistencia

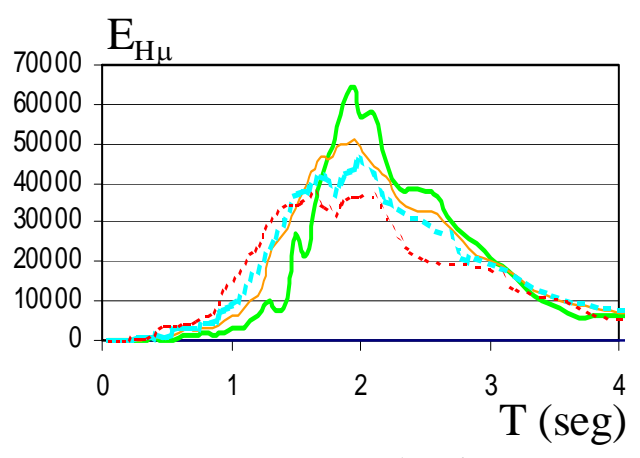

c) Energía plástica

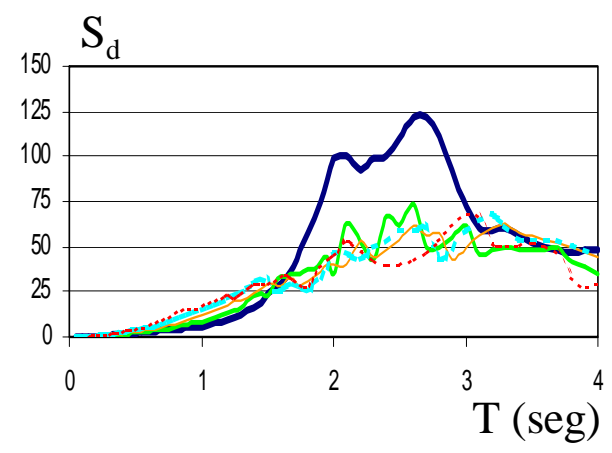

b) Desplazamiento

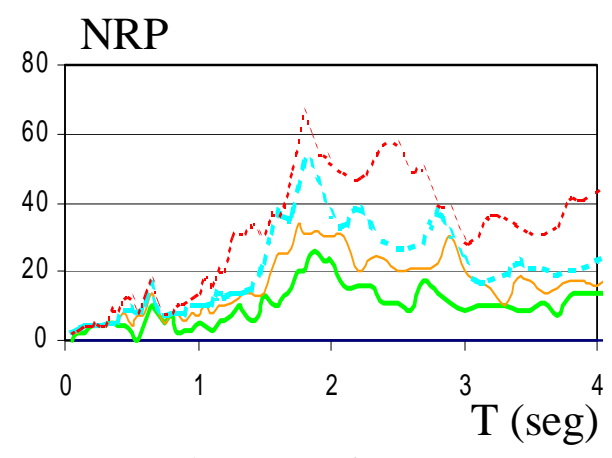

d) Reversiones

Figura 1 Espectros de respuesta para la componente EO de SCT 
Como consecuencia de lo discutido en el párrafo anterior, es posible prever el uso futuro de espectros de diseño correspondientes a diferentes demandas sísmicas, tal como desplazamiento y energía, durante el prediseño y diseño de algunas estructuras sismorresistentes. Dentro de este contexto, resulta conveniente revisar y enfatizar la importancia que tienen las demandas de deformación plástica, tanto máxima como acumulada, en el desempeño estructural, y plantear como pueden considerarse estas demandas dentro de un diseño sísmico por desempeño.

\section{LA ENERGÍA PLÁSTICA COMO UN PARÁMETRO DE DISEÑO}

Una metodología numérica de diseño sísmico requiere relacionar, de una manera confiable, la respuesta de la estructura sismorresistente con el nivel de daño que la misma exhibe después que se le sujeta a la(s) excitación(es) sísmica(s) de diseño. Esto puede lograrse mediante el uso de índices de daño, que son expresiones analíticas que relacionan el valor de una o más demandas sísmicas con el nivel de daño, tanto estructural como no estructural, que una estructura exhibe después de haber estado sujeta a dichas demandas. Terán-Gilmore (1998a) discute el uso de índices de daño para determinar las características mecánicas de una estructura sismorresistente (resistencia, rigidez, capacidad máxima y acumulada de deformación), de manera que su respuesta dinámica quede dentro de límites de respuesta que sean consistentes con el desempeño deseado para la misma.

En lo que se refiere al daño estructural, los índices de daño que se han planteado hasta el momento suelen considerar una medida de las demandas de deformación en la estructura (Williams y Sexsmith, 1995). Mientras que un porcentaje elevado de ellos considera únicamente la demanda de deformación máxima, otros toman en cuenta de alguna manera las demandas acumuladas de deformación plástica (en algunos casos a través de la energía plástica disipada durante la excitación sísmica).

Para plantear el posible uso de la demanda de disipación de energía plástica dentro del contexto del diseño sísmico, primero es necesario discutir el significado físico de dicha energía. Lo primero que puede decirse es que el total de la energía plástica disipada por un sistema durante una excitación sísmica, denotada aquí como $E_{H \mu}$, se incrementa conforme mayores sean sus demandas acumuladas de deformación plástica; y que por tanto, el nivel de daño en ese sistema dado tiende a incrementarse conforme su $E_{H \mu}$ se incrementa. En segundo lugar, es necesario recalcar que $E_{H \mu}$ por sí misma no proporciona toda la información requerida para determinar la magnitud de los ciclos de comportamiento plástico, ni de su secuencia en el tiempo. A pesar de lo último, estudios recientes sugieren que $E_{H \mu}$ puede utilizarse para estimar el daño que se acumula en la estructura como consecuencia del fenómeno de fatiga de bajo ciclaje (Chai, Romstad y Bird, 1992; Terán-Gilmore, 1996; Ávila y Terán-Gilmore, 2000).

El uso directo del valor de la $E_{H \mu}$ puede no aportar información suficiente acerca de la importancia que las demandas acumuladas de deformación plástica tienen en el desempeño sísmico de la estructura. Para ilustrar esto, considere la posibilidad de que la demanda de $E_{H \mu}$ en un sistema con una resistencia muy elevada y que incursiona una sola vez en el rango de comportamiento plástico, resulte mayor que la demanda de $E_{H \mu}$ para un sistema con baja 
resistencia y que incursiona varias veces en el rango de comportamiento plástico. Desde el punto de vista de daño acumulado, el elemento con menor resistencia sufrirá un nivel mayor de daño, no obstante que su demanda de $E_{H \mu}$ es menor. Por tanto, es necesario tomar en cuenta simultáneamente los valores de $E_{H \mu}$, y de la resistencia y rigidez del sistema, para evaluar la importancia de las demandas plásticas acumuladas. Dentro de este contexto, resulta conveniente introducir el concepto de energía plástica normalizada $\left(N E_{H \mu}\right)$ definida como la relación entre la $E_{H \mu} \mathrm{y}$ el producto de la resistencia $\left(F_{y}\right)$ y desplazamiento de fluencia $\left(\delta_{y}\right)$ del sistema.

$$
N E_{H \mu}=\frac{E_{H \mu}}{F_{y} \delta_{y}}
$$

En la Figura 2 se ilustra la $E_{H \mu}$ (igual al área sombreada en la Figura 2a) para un sistema con comportamiento elastoplástico perfecto y sujeto a lo que en este artículo se entenderá como un ciclo de comportamiento plástico. Conforme a lo ilustrado en la Figura 2, se entiende por ciclo de comportamiento plástico una secuencia de deformación que inicia a partir de una fuerza lateral de cero, y que incluye toda la deformación plástica que se acumula en el sistema hasta el punto en que se presenta una reversión de carga que implica fluencia del sistema en sentido opuesto. Note que la secuencia de deformación mostrada en la Figura 2a pudiera ser interpretada por algunos investigadores como un cuarto de ciclo; mientras que aquellas incluidas en las Figuras 2b y 2c, como medio ciclo. Bajo la definición de ciclo planteada para este artículo, las secuencias en estas tres figuras se aceptan (cada una) como un ciclo de deformación plástica. Para un ciclo de deformación plástica (bajo la acepción que aquí se le ha dado), se tiene:

$$
E_{H \mu}=\left(\delta_{i}-\delta_{y}\right) F_{y}
$$

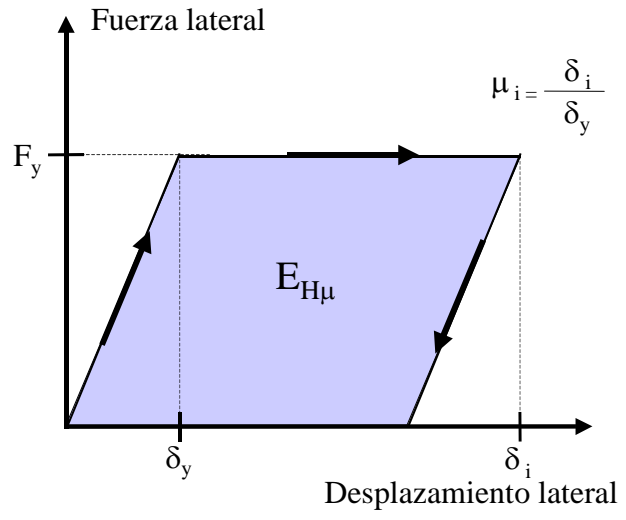

a) Ciclo inicial

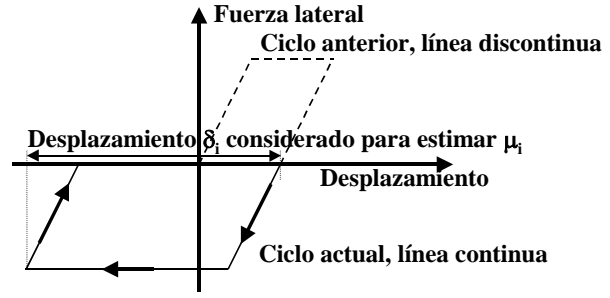

b) Segundo ciclo

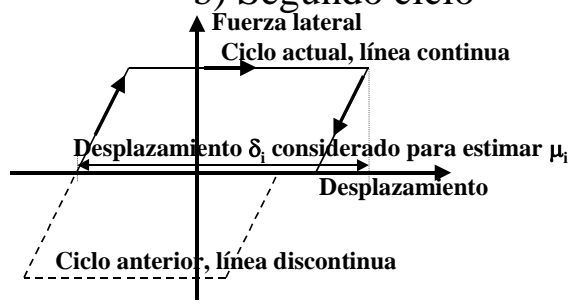

c) Ciclos subsecuentes

Figura 2 Ciclos de comportamiento plástico en sistema elastoplástico perfecto 
La magnitud del ciclo se caracterizará en este artículo por medio de la demanda de ductilidad asociada al mismo, denotada como ductilidad cíclica $\left(\mu_{i}\right)$, y definida como el desplazamiento máximo durante el mismo ( $\delta_{i}$ conforme a lo ilustrado en la Figura 2), normalizado por el desplazamiento de fluencia $\left(\mu_{i}=\delta_{i} / \delta_{y}\right)$. Considerando lo anterior, $E_{H \mu}$ puede expresarse como:

$$
E_{H \mu}=\left(\mu_{i}-1\right) \delta_{y} F_{y}
$$

Combinando las Ecuaciones 1 y 3, puede concluirse que la energía plástica normalizada correspondiente al ciclo puede expresarse como:

$$
N E_{H \mu}=\mu_{i}-1
$$

Para los ciclos ilustrados en la Figura 2, $N E_{H \mu}$ tiene un significado físico muy claro, ya que representa una medida de la ductilidad cíclica; en particular, $N E_{H \mu}$ representa el desplazamiento que sufre la estructura en su rango plástico de comportamiento normalizado por $\delta_{\mathrm{y}}$. Para un sistema elastoplástico perfecto sujeto a deformaciones plásticas acumuladas en dos sentidos opuestos, $N E_{H \mu}$ representa la suma de los desplazamientos plásticos que sufre la estructura durante todos los ciclos de deformación plástica, normalizada por $\delta_{\mathrm{y}}$ :

$$
N E_{H \mu}=\sum_{i=1}^{\text {Nciclos }}\left(\mu_{i}-1\right)
$$

donde Nciclos es el número total de ciclos de comportamiento plástico. Aunque para un sistema con comportamiento histerético degradante, $N E_{H \mu}$ pierde el significado físico que tiene para un sistema elastoplástico perfecto, el manejo juicioso de $N E_{H \mu}$ permite evaluar la importancia de las demandas acumuladas de deformación plástica en el desempeño estructural.

Uno de los índices de daño más utilizados para evaluar el daño estructural en elementos de concreto reforzado es el propuesto por Park y Ang (1985), que plantea que el nivel de daño puede estimarse como la suma de aquel producido por la demanda máxima de deformación más aquel producto de las demandas acumuladas de deformación plástica (representadas por medio de la energía plástica):

$$
I D_{P A}=\frac{\delta_{\max }}{\delta_{u}}+\beta \frac{\int d E_{H \mu}}{F_{y} \delta_{u}}
$$

donde $\delta_{\max }$ es la demanda máxima de deformación durante el sismo, $\delta_{u}$ es la capacidad última de deformación del elemento, $d E_{H \mu}$ es un diferencial de energía plástica y $\beta$ es una constante que toma en cuenta el efecto de las demandas acumuladas de deformación plástica en el nivel de daño del elemento de concreto. La integral $\int d E_{H \mu}$ evaluada para la duración total de la excitación sísmica es igual a $E_{H \mu}$. Un $I D_{P A}$ menor que 0.4 implica daño reparable; de 0.4 a 1.0 , daño no reparable; y mayor que 1.0, falla del elemento. Cosenza et al. (1990) han observado que un $\beta$ igual a 0.15 resulta en que el índice de Park y Ang arroje resultados cercanos al que arrojan otros índices de daño. Vale la pena destacar que el índice de Park y Ang contempla la normalización de $E_{H \mu}$ por el producto $\mathrm{F}_{\mathrm{y}} \delta_{\mathrm{u}}$. 
A pesar de que el índice de Park y Ang no toma en cuenta la forma en que se disipa la energía plástica durante la excitación sísmica, es uno de los índices de daño mas empleados, debido a su sencillez, para evaluar daño en elementos y estructuras de concreto reforzado. Acorde a lo planteado por Terán-Gilmore et al. (1998), el índice de Park y Ang puede plantearse conforme a lo siguiente:

$$
I D_{P A}=\frac{\mu_{\delta \max }}{\mu_{\delta u}}+\beta \frac{N E_{H \mu}}{\mu_{\delta u}}
$$

donde $\mu_{\delta \max }$ es la demanda máxima de ductilidad durante el sismo y $\mu_{\delta u}$ es la ductilidad última que el elemento o estructura pueden alcanzar bajo un estado de deformación monotonicamente creciente. La Ecuación 7 muestra que el nivel de daño acorde al índice de Park y Ang puede expresarse como una combinación lineal de las demandas máxima $\left(\mu_{\delta \text { max }}\right)$ y acumulada $\left(N E_{H \mu}\right)$ de deformación plástica en la estructura.

Con un enfoque diferente, derivado directamente del concepto de fatiga de bajo ciclaje, se ha planteado la hipótesis de Miner. En este artículo la hipótesis de Miner se planteará directamente como un índice de daño, $I D_{M H}$, conforme lo siguiente:

$I D_{M H}=\sum_{i=1}^{\text {Nciclos }} \frac{n_{i}}{N_{i}}$

donde $N_{i}$ representa el número de ciclos de comportamiento plástico con ductilidad cíclica igual a $\mu_{i}$ (bajo la acepción de ciclo planteada en este artículo) que la estructura puede soportar antes de fallar por fatiga de bajo ciclaje; y $n_{i}$ el número de ciclos de comportamiento plástico con ductilidad cíclica $\mu_{i}$ inducidos en la estructura durante la excitación sísmica. En su formulación tradicional, $I D_{M H}$ igual o mayor que 1 implica la falla del elemento o sistema estructural. Aunque la hipótesis de Miner no toma en cuenta la secuencia en que ocurren los ciclos de deformación plástica en la estructura, si hace consideraciones acerca de cómo se ha disipado la energía plástica. En particular $I D_{M H}$ considera, a través de los valores de $N_{i}$, que conforme se incrementa su $\mu_{i}$, el número de ciclos de comportamiento plástico que la estructura es capaz de soportar se reduce de manera importante. En este artículo, $N_{i}$ se definirá a partir del criterio planteado por Chen y Gong (1986), que se ilustra en la Figura 3a y se resume a continuación:

$(\mu-0.676)(M-0.676)=1.403$

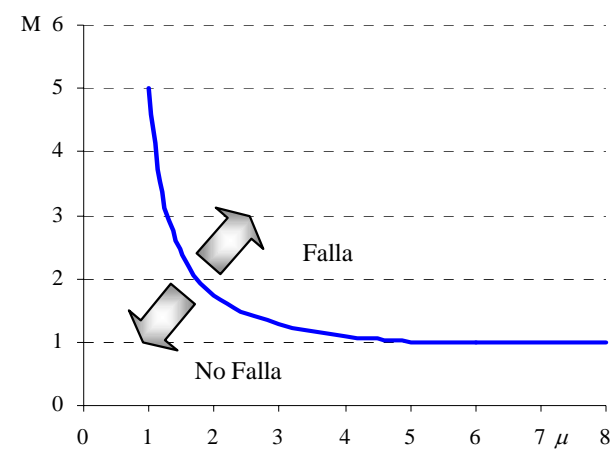

a) Criterio de Chen y Gong

Figura 3 Distribución del número de ciclos de comportamiento plástico 
El criterio de Chen y Gong considera simultáneamente medidas de las demandas máxima y acumulada de deformación plástica ( $\mu$ y $M$, respectivamente). El valor de $M$ correspondiente a un ciclo con ductilidad $\mu_{i}$ se define como:

$$
M_{i}=\frac{E_{H \mu i}}{F_{y} \delta_{y}\left(\mu_{\delta u}-1\right)}
$$

donde $M_{i}$ es el valor de $M$ correspondiente a ciclos de comportamiento plástico con magnitud $\mu_{i}$; $E_{H \mu i}$ es la energía plástica total que la estructura puede disipar cuando se le lleva a la falla por medio de inducirle exclusivamente ciclos de comportamiento plástico con magnitud $\mu_{i}$. Si se normaliza el valor de $E_{H \mu i}$ por la energía plástica disipada durante un ciclo de comportamiento plástico con magnitud $\mu_{i}$, se obtiene el número total de ciclos de magnitud $\mu_{i}$ que la estructura puede tolerar antes de fallar. Si se considera que la energía plástica disipada durante un ciclo de comportamiento plástico con magnitud $\mu_{i}$ esta dada por la Ecuación 3, entonces:

$$
N_{i}=\frac{E_{H \mu i}}{F_{y} \delta_{y}\left(\mu_{i}-1\right)}
$$

Despejando $E_{H \mu i}$ de la Ecuación 10 y sustituyendo en la Ecuación 11 se tiene que:

$$
N_{i}=\frac{\left(\mu_{\delta u}-1\right)}{\left(\mu_{i}-1\right)} M_{i}
$$

Finalmente, sustituyendo la Ecuación 12 en la Ecuación 9:

$$
N_{i}=\left(\frac{1.403}{\mu_{i}-0.676}+0.676\right) \frac{\mu_{\delta u}-1}{\mu_{i}-1}
$$

Los valores de $N_{i}$ planteados a partir del criterio de Chen y Gong se resumen en la Figura 3 b, donde se muestra que el número de ciclos de comportamiento plástico que una estructura es capaz de soportar crece considerablemente si la demanda de ductilidad asociada a estos ciclos se reduce.

La hipótesis de Miner considera que el daño inducido en la estructura en cada ciclo de comportamiento plástico es independiente al daño producido en cualquier otro ciclo; de manera que debe establecerse una convención clara para definir y delimitar cada uno de estos ciclos. Varios investigadores han adoptado diferentes definiciones de ciclo de comportamiento plástico, y es difícil plantear que haya una manera única y correcta para hacer esto. Quizá la manera más inmediata para contabilizar dichos ciclos sea delimitarlos de acuerdo a la secuencia en que ocurren durante la excitación sísmica, tal como se sugiere en la Figura 4b. Sin embargo, existen ciertos casos, como el ilustrado en la Figura 4a, donde dos o más ciclos de deformación plástica baja o moderada dan lugar a un ciclo de mayor magnitud. En este caso, el daño producido por cada uno de los dos ciclos no puede considerarse independiente del ocurrido en el otro, de tal manera que el daño ocurrido en la estructura puede subestimarse de manera importante si los ciclos se delimitan estrictamente de acuerdo a su secuencia de ocurrencia. En casos como este, algunos investigadores han recurrido a planteamientos como el de la gota de lluvia (rainflow en inglés), ilustrado en la Figura 4c y explicado en detalle en Nassar y Krawinkler (1991), para delimitar los ciclos de comportamiento plástico. Cabe aclarar que, como se discutirá en detalle 
más adelante, la aplicación del planteamiento rainflow (o de cualquier otro) no elimina todas las inconsistencias en cuanto a la delimitación y contabilización de los ciclos de comportamiento plástico.

Hasta ahora se han discutido dos índices de daño que, aunque consideran las demandas acumuladas de deformación plástica, se basan en enfoques significativamente diferentes. Por un lado, $I D_{P A}$ tiene como ventaja su sencillez, lo que hace posible su uso en aplicaciones prácticas (Bertero y Bertero, 1992; Terán-Gilmore, 1996). Sin embargo, el uso de este índice puede llegar a ser cuestionable, ya que ignora la manera en que se disipa la energía plástica en la estructura (esto es, la secuencia y magnitud de los ciclos de deformación plástica). Otros inconvenientes del uso de $I D_{P A}$ se discuten en Terán-Gilmore et al. (1998). Por el otro lado, $I D_{M H}$ tiene la ventaja de considerar el número y la magnitud de los ciclos de deformación plástica, lo que hace posible una mejor estimación del nivel de daño en elementos o estructuras sujetas a secuencias "extremas” de acumulación de deformaciones plásticas. Como secuencia "extrema” se entiende una secuencia donde la gran mayoría o la totalidad de la energía plástica se disipa en ciclos de comportamiento plástico con $\mu_{i}$ muy pequeña o muy grande. El $I D_{M H}$ no toma en cuenta la secuencia de los ciclos de comportamiento plástico, la que puede llegar a ser importante para definir el patrón de daños que exhibe un sistema estructural. Otro inconveniente de $I D_{M H}$ es que su aplicación práctica se dificulta debido a la gran cantidad de información que su planteamiento requiere.

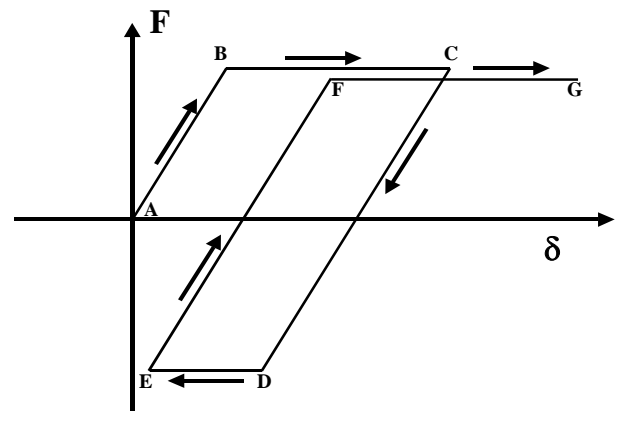

a) Secuencia real de ciclos

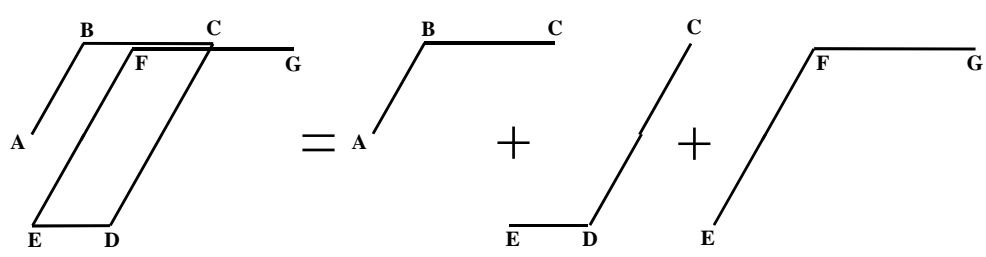

b) Contabilización de ciclos acorde a su ocurrencia

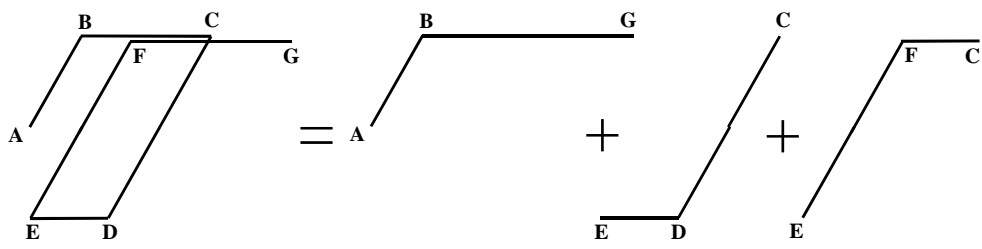

C) Contabilización de ciclos con criterio rainflow

Figura 4 Ilustración del método de la gota de lluvia 
Al contrastar las ventajas y desventajas de $I D_{P A}$ y $I D_{M H}$, surge la inquietud acerca de cuando son importantes el número y magnitud de los ciclos de comportamiento plástico en el desempeño sísmico de las estructuras. Considere el caso en que $n_{i}$ y $N_{i}$ puedan relacionarse, para toda $\mu_{i}$, mediante la misma constante de proporcionalidad:

$$
n_{i}=\alpha N_{i}
$$

donde $\alpha$ representa la constante de proporcionalidad. Sustituyendo la Ecuación 14 en la 8, se tiene que:

$$
D M I_{M H}=\sum_{i=1}^{\text {Nciclos }} \frac{n_{i}}{N_{i}}=\sum_{i=1}^{\text {Nciclos }} \frac{\alpha N_{i}}{N_{i}}
$$

Note que en el lado derecho de la Ecuación 15, el valor de $N_{i}$ se cancela para cada uno de los términos de la sumatoria, de tal manera que bajo el caso planteado por la Ecuación 14, no es necesario conocer con precisión el número y la magnitud de los diferentes ciclos de deformación plástica. Bajo estas circunstancias, sería posible plantear una metodología numérica de diseño sísmico, que considere exclusivamente la demanda de energía plástica en la estructura sismorresistente. En otras palabras, podría considerarse el uso de índices similares al de Park y Ang para plantear, durante el diseño sísmico, el control del daño estructural.

Cabe aclarar que el uso de métodos como el rainflow para delimitar y contar los ciclos de comportamiento plástico no elimina todas las inconsistencias que pueden surgir durante el planteamiento del índice $I D_{M H}$. Por ejemplo, la Figura 5 muestra dos ciclos con la misma magnitud $\mu_{i}=\delta_{i} / \delta_{y}$ (suponiendo que en ambas figuras $\delta_{y}$ es igual). Aunque según lo planteado hasta ahora ambos ciclos producirían el mismo daño en el sistema estructural, una reflexión al respecto nos llevaría a concluir que el ciclo en la Figura 5a tiende a producir mas daño estructural que el mostrado en la Figura 5b. Otro problema, este originado a partir de la definición de ciclo de comportamiento plástico adoptada en este artículo, es que se equipara al ciclo inicial de comportamiento plástico (como el ilustrado en la Figura 2a) con todos los demás ciclos subsecuentes (ilustrados en las Figuras $2 \mathrm{~b}$ y $2 \mathrm{c}$ ). A pesar de los problemas discutidos en este párrafo en cuanto a la delimitación y contabilización de los ciclos de comportamiento plástico, los resultados presentados en este artículo muestran tendencias bien definidas, de tal manera que un cambio en las definiciones y convenciones adoptadas podrían llegar a matizar, pero no a cambiar las conclusiones obtenidas.

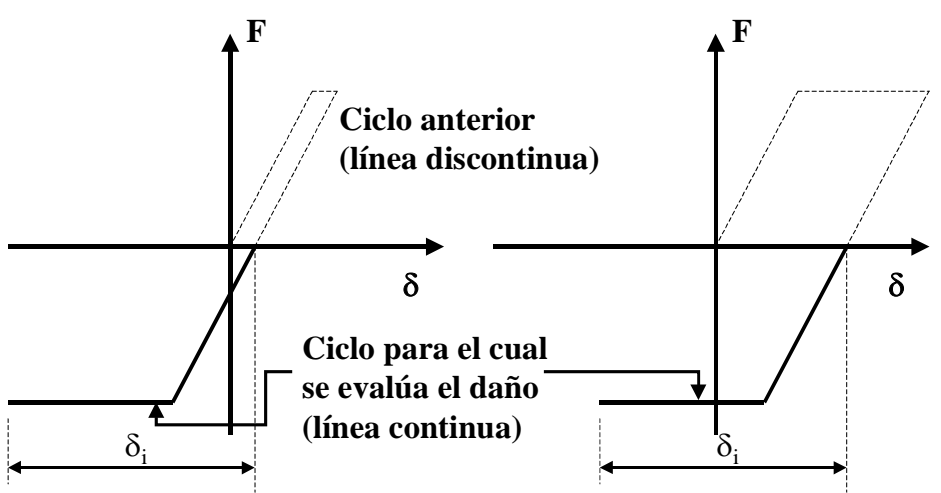

a) Mayor daño

b) Menor daño

Figura 5 Ciclos de misma magnitud asociado a diferentes niveles de daño 


\section{IMPORTANCIA DEL NÚMERO Y MAGNITUD DE CICLOS PLÁSTICOS}

Para los estudios aquí presentados, se utilizaron dos familias de 20 acelerogramas sintéticos, la componente NS de El Centro 1940 y la componente EO de SCT 1985. Los acelerogramas sintéticos fueron generados filtrando ruidos blancos gaussianos con un filtro Kanai-Tajimi (Tajimi 1960). El uso de este filtro implica la definición de los parámetros $T_{g}$ y $\xi_{g}$, que están relacionados con el periodo predominante del terreno y con el contenido de frecuencias de la excitación (Lai 1982, Tung et al. 1992). La Tabla 1 muestra el valor asignado a los parámetros del filtro Kanai-Tajimi para la generación de las dos familias de acelerogramas sintéticos. Cabe aclarar que se eliminaron las frecuencias bajas de las muestras por medio del filtro de Hodder (1983), y que dichas muestras se filtraron en el dominio del tiempo de acuerdo a las indicaciones de Tung et al. (1992), de manera de suministrarles una variación razonable en el tiempo de la intensidad sísmica. Los resultados que aquí se presentan se obtuvieron para sismos sintéticos con duración de fase intensa, definida acorde a Trifunac y Brady (1975), de 30 segundos. Este valor es característico de movimientos sísmicos intensos de alta duración (por ejemplo, según esta definición el Centro NS tiene una duración cercana a los 25 segundos; mientras que el valor correspondiente al SCT EO es de 35 segundos). Cabe aclarar que las tendencias observadas para acelerogramas sintéticos con duraciones de 10 y 20 segundos son similares a las discutidas mas adelante para los movimientos de 30 segundos. Los detalles de la generación de los acelerogramas puede encontrarse en Terán-Gilmore (1996).

Tabla 1. Parámetros del filtro Kanai-Tajimi.

\begin{tabular}{|c|c|c|}
\hline Tipo de terreno & $\mathrm{T}_{\mathrm{g}}(\mathrm{s})$ & $\xi_{\mathrm{g}}$ \\
\hline Firme & 0.4 & 0.35 \\
\hline Blando & 2.0 & 0.05 \\
\hline
\end{tabular}

La Figura 6 compara, para un $\xi$ de 0.05 , el promedio de los 20 espectros de resistencia elásticos de las familias de acelerogramas sintéticos con aquellos obtenidos para El Centro NS 1940 y SCT EO 1985.

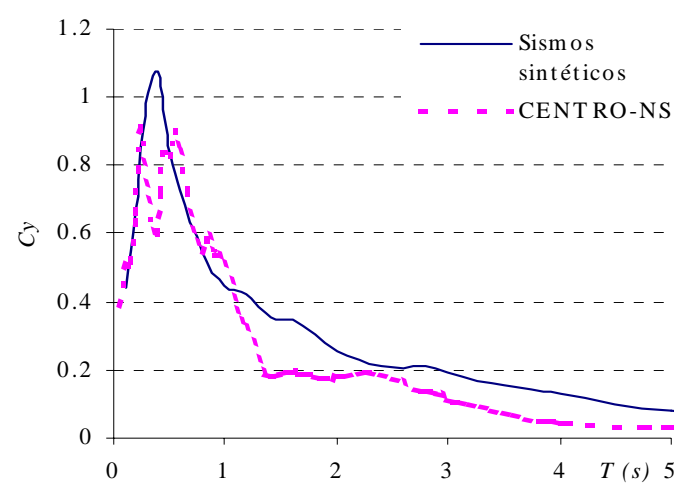

a) Suelo firme

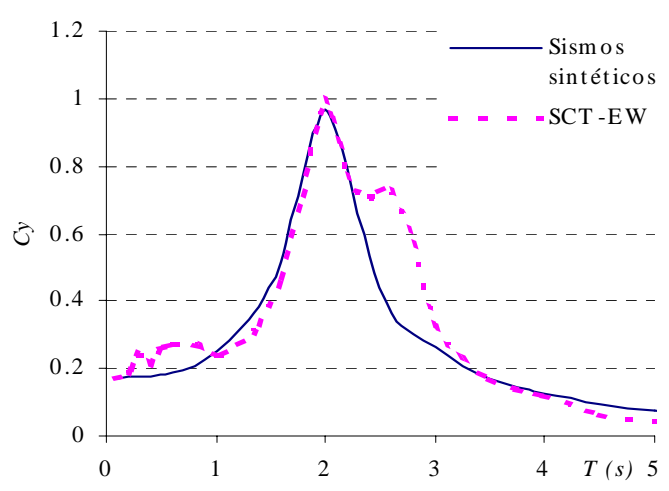

b) Suelo blando

Figura 6. Espectros de resistencia elásticos para los acelerogramas utilizados $(\xi=0.05)$ 
El estudio aquí presentado se llevo a cabo a partir de la respuesta de sistemas de un grado de libertad (S1GL) con comportamiento elastoplástico perfecto. La respuesta de los S1GL se estimó con el método de Newmark con aceleración constante. Las características mecánicas de los S1GL se asignaron conforme a lo siguiente:

- Se manejaron periodos (T) que abarcan desde 0.4 hasta 4.0 seg, con lo que se consideró un rango de $T$ que abarca las propiedades dinámicas de la gran mayoría de estructuras existentes.

- Se consideraron coeficientes equivalentes de amortiguamiento ( $\xi$ ) de 0.05 y 0.20 , que se consideraron valores característicos de sistemas estructurales tradicionales y de sistemas pasivos de disipación de energía, respectivamente.

- Se consideraron demandas máxima de ductilidad $(\mu)$ de 2, 3 y 4, que representan un rango de demandas suficientemente amplio para tomar en cuenta la respuesta dinámica de estructuras no dúctiles y dúctiles. Se aclara mediante la Figura 7 que la demanda máxima de ductilidad $(\mu)$ que sufre un sistema durante una excitación sísmica, no necesariamente coincide con la demanda máxima de ductilidad que puede ocurrir en el ciclo de comportamiento plástico de mayor amplitud $\left(\mu_{\text {imax }}\right)$. Conforme a lo mostrado, $\mu_{\text {imax }}$ puede alcanzar valores hasta de $2 \mu-1$.

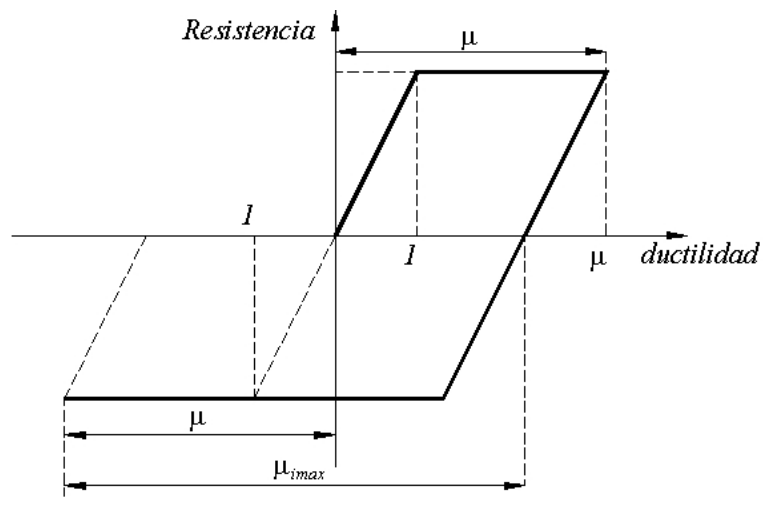

Figura 7 Comparación entre ductilidad máxima $(\mu)$ y ductilidad cíclica máxima $\left(\mu_{\text {imax }}\right)$

Una vez estimada la respuesta de los S1GL, se utilizó el método de la gota de lluvia para contar el número de ciclos $n_{i}$ correspondiente a diferentes rangos de valores de $\mu_{i}$. Cada una de las gráficas presentadas en la Figura 8 presenta los $n_{i}$ que corresponden a diferentes rangos de $\mu_{i}$ para S1GL que desarrollan una ductilidad máxima de 4. Note que a partir de la Figura 7 puede explicarse porque el rango superior de $\mu_{i}$ comprende valores entre 6.38 y 6.92 mientras que $\mu$ es igual a 4. Los rangos de valores de $\mu_{i}$ contemplados en las figuras se definieron de tal manera que se contara con diez rangos uniformes de $\mu_{i}$ que abarcaran desde un valor mínimo de uno hasta el valor máximo encontrado en los análisis paso a paso (que resultó igual a 6.92).

Las Figuras 8a a 8d corresponden a excitaciones sísmicas características de suelo firme; mientras que las Figuras 8e y 8f, a excitaciones características de suelo blando. En la Figura 8 no se presenta el número promedio de ciclos en los S1GL, sino el acumulado de las 20 excitaciones sísmicas.

Para todas las graficas presentadas en la Figura 8, puede observarse que el valor $n_{i}$ se incrementa con un decremento del valor de $\mu_{i}$, tendencia particularmente notoria conforme $\mu_{i}$ 
tiende a 1. La comparación de las Figuras 8 y 3b sugieren que, con excepción de la Figura 8f, $n_{i}$ y $N_{i}$ tienen una dependencia similar con respecto al valor de $\mu_{i}$.

Comparando la Figura 8a con la Figura 8b, ambas obtenidas para $\xi$ de 0.05 y suelo firme, puede concluirse que conforme el valor del $T$ crece, los S1GL tienden a disipar un mayor porcentaje de la energía plástica en ciclos de mayor amplitud. Esto se concluye porque en la Figura 8b, obtenida para $T$ de 2 seg, los valores de $n_{i}$ para $\mu_{i}$ alta son más importantes, relativos a los valores de $n_{i}$ para $\mu_{i}$ baja, que en el caso de la Figura 8a, obtenida para un $T$ de 0.4 seg. Por ejemplo, considere que en la Figura 8b se tiene un $n_{i}$ de 44 para $\mu_{i}$ de 3.37 a 3.96, mientras que el $n_{i}$ correspondiente a $\mu_{i}$ de 1 a 1.59 es de 478; esto implica una relación (44/478) de 0.0921. Los valores correspondientes en la Figura 8a son 51 y 1536, que dan una relación de 0.0332.

La comparación de las Figuras 8a y 8c, así como de las Figuras 8b y 8d, ilustran el efecto de un incremento del $\xi$ en el valor de $n_{i}$. Puede concluirse que, aunque un incremento de $\xi$ se refleja en una disminución importante del valor de $n_{i}$, las tendencias relativas observadas para valores de $\xi$ de 0.05 y 0.20 son muy similares. Quizá pueda mencionarse que un incremento de $\xi$ resulta en que un porcentaje ligeramente mayor de la energía plástica se disipe en ciclos de mayor amplitud.

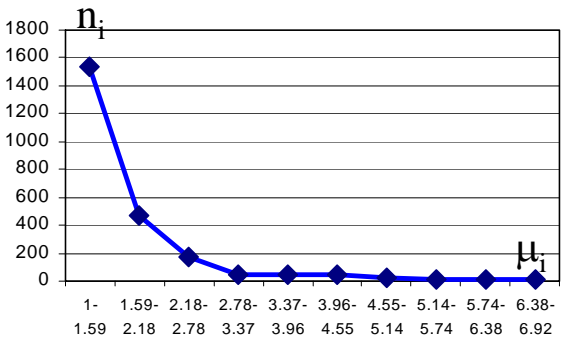

a) Suelo firme, $\mathrm{T}=0.4 \operatorname{seg}$ y $\xi=0.05$

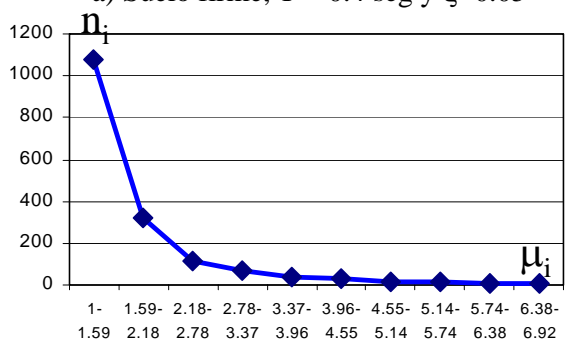

c) Suelo firme, $\mathrm{T}=0.4 \operatorname{seg}$ y $\xi=0.20$

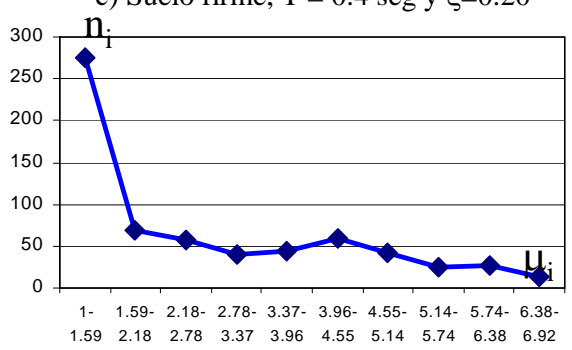

e) Suelo blando, $\mathrm{T}=0.8 \operatorname{seg}$ y $\xi=0.05$

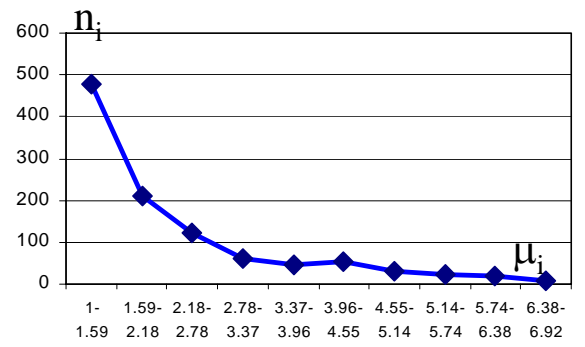

b) Suelo firme, $\mathrm{T}=2.0 \mathrm{seg}$ y $\xi=0.05$

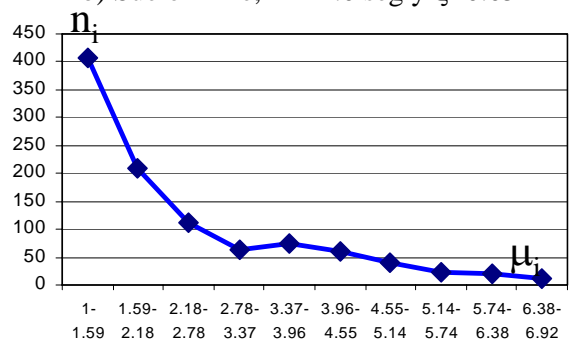

d) Suelo firme, $\mathrm{T}=2.0 \mathrm{seg}$ y $\xi=0.20$

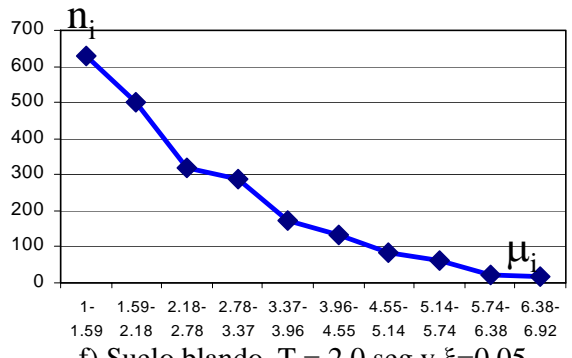

f) Suelo blando, $T=2.0 \operatorname{seg}$ y $\xi=0.05$

Figura 8 Demanda real de ciclos de comportamiento plástico 
Las Figuras 8e y $8 \mathrm{f}$ muestran resultados de S1GL con los mismos $T$ y $\xi$ que las Figuras 8a y 8 b, respectivamente, pero ahora sujetos a excitaciones típicas de suelo blando. A partir de lo ilustrado, puede concluirse que para suelo blando, los S1GL tienden a disipar un mayor porcentaje de la energía plástica en ciclos de mayor amplitud que los observados en suelo firme, siendo esto particularmente notorio para $T$ cercano a $T_{g}$ de la excitación de suelo blando, que en este caso es igual a 2 seg.

Con el fin de estudiar si los valores de $n_{i}$ y $N_{i}$ siguen una relación similar a la planteada por la Ecuación 14, la Figura 9 hace una comparación directa de las tendencias para $n_{i}$ ilustradas en la Figura 8 y los valores de $N_{i}$ obtenidos con el criterio de Chen y Gong (Figura 3b). Para facilitar la comparación de las tendencias, las curvas de $n_{i}$ vs. $\mu_{i}$ y de $N_{i}$ vs. $\mu_{i}$ se normalizaron de manera que tanto su abcisa como ordenada máximas, sean iguales a 1 . Se aclara que la comparación que se establece es cualitativa, y se reconoce que la normalización planteada debe refinarse si se persiguen fines cuantitativos.

La Figura 9a fue obtenida a partir de la respuesta de S1GL con $\xi$ de 0.05 sujetos a excitaciones sintéticas de suelo firme. Puede notarse que para un amplio rango de valores de $T$, la dependencia de $n_{i}$ normalizada con respecto al valor de $\mu_{i}$ normalizado es muy similar a la observada para el $N_{i}$ normalizado. Puede notarse que conforme el valor de T crece, un mayor porcentaje de la energía plástica tiende a disiparse en ciclos de mayor amplitud. Dada la similitud entre las curvas de $n_{i}$ normalizado y $N_{i}$ normalizado, puede decirse que la Ecuación 14 es una aproximación razonable a la relación existente entre $n_{i}$ y $N_{i}$ y que, por tanto, con excepción de S1GL con $T$ alto, la manera en que se disipa la energía plástica en S1GL sujetos a excitaciones típicas de suelo firme no tiene relevancia en la predicción de su nivel de daño estructural. En el caso de $T$ alto, un índice de daño que no considere la forma en que se ha disipado la energía plástica tendería a subestimar ligeramente el nivel de daño estructural. Sin embargo, es importante recordar que las demandas de energía plástica, inducidas por excitaciones típicas de suelo firme en estructuras con $T$ alto, son de bajas a muy bajas, lo que sugiere que en este rango de $T$ no se incurrirá en errores significativos si se ignora la manera en que se disipa la energía plástica.

Ávila y Terán-Gilmore (2000) han observado que para $T$ bajo, $\xi$ tiene poco efecto en las tendencias descritas en el párrafo anterior, mientras que para $T$ moderado y alto, un incremento de $\xi$ resulta en que un porcentaje mayor de la energía plástica tienda a disiparse en ciclos de mayor amplitud. Esto implica que la ligera subestimación del daño estructural discutida en el párrafo anterior se presenta a partir de valores moderados de $T$.

La Figura 9b fue obtenida a partir de la respuesta de S1GL con $\xi$ de 0.05 sujetos a excitaciones típicas de suelo blando. Puede notarse que el valor de $n_{i}$ normalizado suele ser significativamente mayor, para valores intermedios y altos de $\mu_{i}$ normalizada, que el valor de $N_{i}$ normalizado; diferencia que tiende a ser más importante conforme el valor de $T$ se acerca al valor de $T_{g}$. Por tanto, la subestimación de la cual se hablo para $T$ alto en el caso de suelo firme, se da prácticamente en todo el rango de $T$ estudiado (aunque de manera más notoria para $T$ cercano a $T_{g}$ ) en el caso de excitaciones características de suelo blando. Es importante recordar que las demandas de energía plástica, inducidas por excitaciones típicas de suelo blando, disminuyen considerablemente conforme el $T$ de la estructura se aleja de $T_{g}$. Lo anterior sugiere que solo para 
estructuras con $T$ cercano a $T_{g}$ se incurrirá en errores significativos si se ignora la manera en que se disipa la energía plástica. Ávila y Terán-Gilmore (2000) han observado que un incremento en $\xi$ tiene poco efecto en las tendencias descritas en este párrafo; acaso pueda decirse que un incremento de $\xi$ suaviza las tendencias observadas para $T$ bajo.

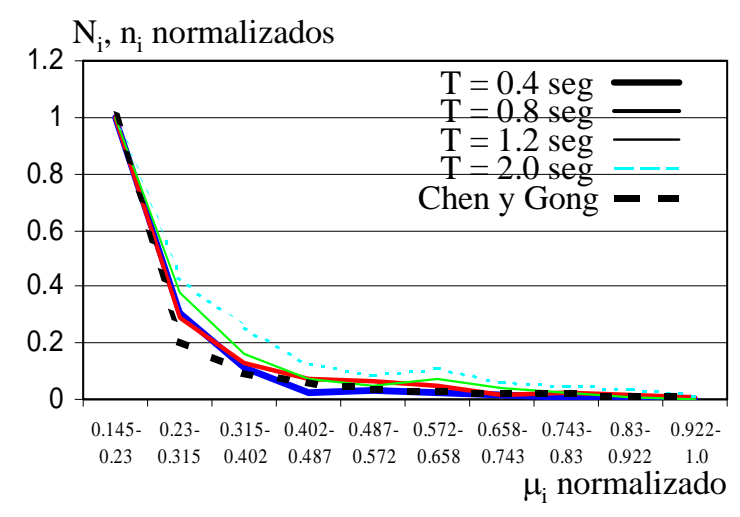

a) Suelo firme

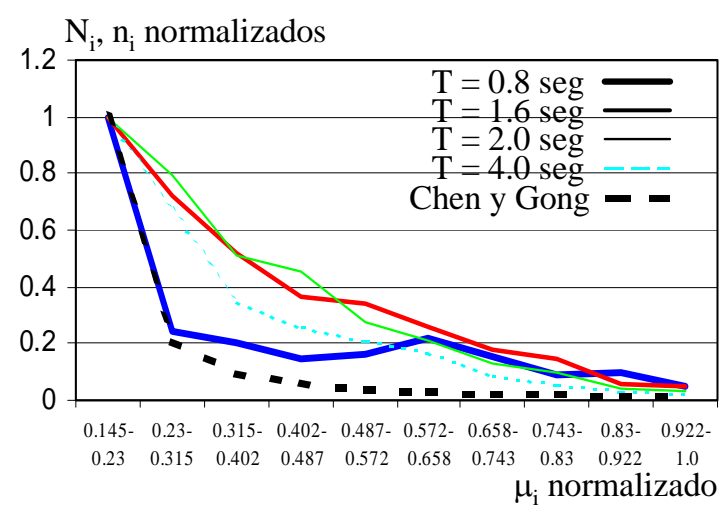

b) Suelo blando

Figura 9 Distribución normalizada de ciclos para $\xi=0.05$

La Figura 10 resume e ilustra el impacto que la historia de disipación de energía tiene en la estimación del daño estructural. Para ello, la Figura 10 presenta espectros de resistencia de daño constante y $\xi$ de 0.05 , que se caracterizan por indicar, para los diferentes valores de $T$, la resistencia mínima que deberían tener los S1GL para que su nivel de daño después de la excitación sísmica corresponda a colapso incipiente. Se presentan dos espectros de daño constante: uno estimado a partir de que $I D_{M H}=1$ con $N_{i}$ estimado a partir de la Figura 3b; y otro que corresponde a $I D_{P A}=1$ considerando que $\mu_{\delta u}$ es igual a 4 .

Note en la Figura 10a, que corresponde al Centro NS, que existen pocas diferencias entre ambos espectros de daño constante, lo que sugiere que para excitaciones características de suelo firme, la forma en que se disipa la energía plástica tiene poca consecuencia en la estimación del daño estructural. En la Figura 10b, correspondiente a SCT EO, puede notarse que el uso de $I D_{P A}$ para establecer el espectro de resistencia correspondiente a daño constante tiende a subestimar, con respecto a lo indicado por el espectro asociado a $I D_{M H}$, la resistencia requerida en los S1GL. Note que esta subestimación es más notoria para $T$ cercano a $T_{g}$, donde alcanza valores entre 15 y $20 \%$ de los predichos por $I D_{M H}$; y que dicha subestimación practicamente desaparece para valores de $T$ lejanos de $T_{g}$. Puede concluirse que para el caso de excitaciones características de suelo blando, la resistencia de diseño obtenida a partir del uso de $I D_{P A}$ debe incrementarse para estructuras con $T$ cercano a $T_{g}$; este incremento debe reducirse paulatinamente hasta hacerse cero para sistemas con $T$ lejano a $T_{g}$. 


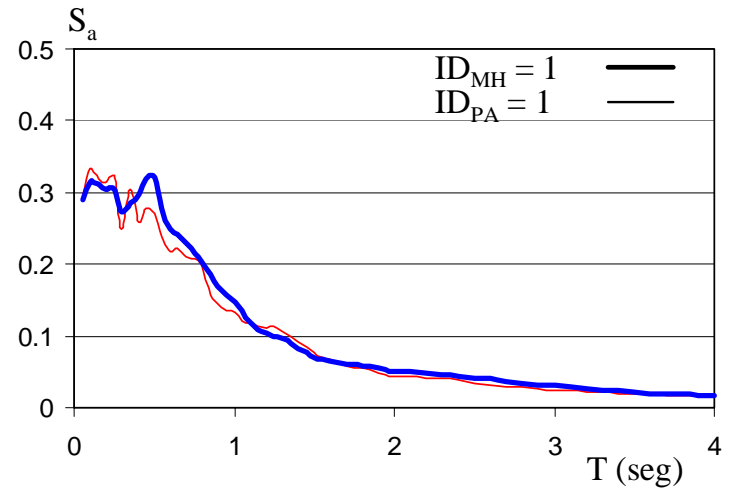

a) El Centro NS

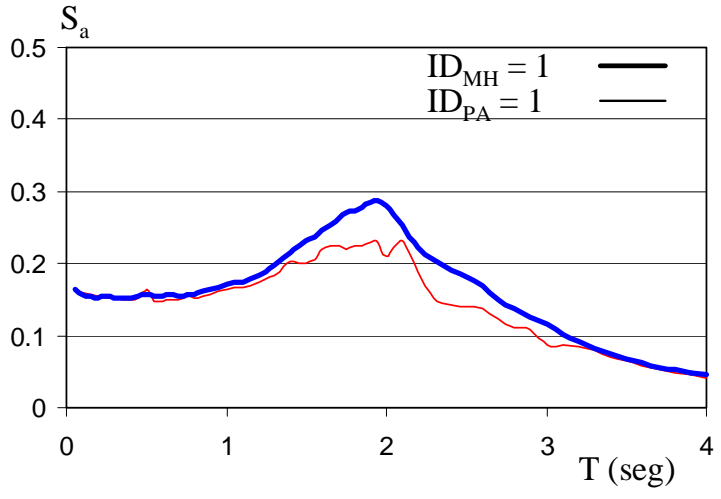

b) SCT EO

Figura 10 Espectros de resistencia para daño constante, $\xi=0.05$

\section{LA ENERGÍA PLÁSTICA EN SISTEMAS DE VARIOS GRADOS DE LIBERTAD}

Una vez establecida la importancia de la historia de disipación de energía plástica en S1GL, es necesario estudiar si la información obtenida a partir de la respuesta de estos sistemas puede utilizarse para establecer el control de daño estructural en sistemas más complejos que posean varios grados de libertad. Varios autores han señalado la posibilidad de predecir el comportamiento de marcos momentorresistentes, particularmente su demanda global máxima de desplazamiento lateral, a partir de modelos equivalentes de un grado de libertad (Qi y Moehle, 1991; Fajfar y Fischinger, 1992). Conforme a lo planteado por dichos autores, el paso inicial para establecer uno de estos modelos consiste en sujetar al marco momentorresistente a un análisis no lineal bajo deformación lateral monotonicamente creciente (tipo pushover), hasta que se alcance un desplazamiento lateral de azotea cercano al esperado durante la excitación sísmica de diseño. En una segunda etapa, se plantea un modo de vibrar que considere la distribución de desplazamiento lateral en altura asociada al desplazamiento de azotea de interés; a partir del cual se estima un factor de participación que permite el planteamiento de un modelo equivalente de un grado de libertad. Note que el planteamiento de este modelo implica el conocimiento de las características mecánicas locales (resistencia y rigidez) del sistema de varios grados de libertad, y la realización de un análisis estático no lineal.

Dentro del contexto planteado por el párrafo anterior, se puede discutir la posibilidad de obtener información útil para la concepción y el diseño sísmico de marcos momentorresistentes regulares a partir de la información contenida en un espectro de respuesta (que se establece a partir de la respuesta de S1GL). Note que dentro del contexto de la normatividad actual, el conocimiento detallado de las características mecánicas locales de las estructuras es un producto final de proceso de diseño sísmico, no información disponible al inicio del mismo (en otras palabras, no existe de salida información para plantear un sistema equivalente de un grado de libertad); y que no resultaría deseable plantear desde el inicio del proceso de diseño sísmico una serie de análisis estáticos no lineales de la estructura. Complementando los dos contextos discutidos hasta el momento, puede decirse que la concepción y el diseño sísmico racional de una estructura requiere del uso de herramientas y métodos simples, basados en la estimación de las demandas sísmicas en la estructura a partir de la respuesta de sistemas sencillos (como es el caso de un S1GL); quedando la 
opción del uso de análisis no lineales para revisar si el producto final del diseño satisface las condiciones de desempeño para las cuales se diseña.

Una de las herramientas más poderosas con las que actualmente cuenta el ingeniero estructural durante el diseño sísmico es el método de análisis estático (especificado en la gran mayoría de códigos de diseño sísmico a nivel mundial). Este método se ha planteado con el fin de hacer posible el diseño sísmico de las estructuras sin la necesidad de llevar a cabo un análisis dinámico completo que implique el cálculo de modos de vibrar, de factores de participación, etc. El método estático asume que la respuesta esta dominada por el primer modo de vibrar, suposición que se ve reflejada, entre otras cosas, en la distribución en altura de las fuerzas laterales de diseño. Además, bajo las consideraciones hechas por este método, el cortante basal de diseño es igual al producto del peso total de la estructura por la ordenada leída directamente del espectro de diseño de resistencia. Cabe aclarar que por lo general, dicha ordenada es función del periodo fundamental de vibración de la estructura, de tal manera que se requiere una estimación de dicho periodo.

En este artículo se plantea la posibilidad de extender el concepto de análisis estático para considerar aquellas demandas sísmicas que, como el desplazamiento lateral máximo y la energía plástica disipada, permiten plantear el control de daño estructural en las estructuras sismorresistentes. En particular, se estudia la pertinencia de utilizar los valores leídos directamente de espectros de respuesta durante una concepción y un diseño sísmico que contemple el efecto de fatiga de bajo ciclaje (sin que se llegue a plantear un análisis dinámico formal que implique la determinación de modos de vibrar y factores de participación). Dentro de este contexto, la única característica dinámica que debe considerarse al principio del proceso de diseño es el periodo fundamental de vibración de la estructura, que permitirá entrar a los diferentes espectros de respuesta para determinar sus demandas sísmicas.

Para explicar lo que se pretende en este artículo, considere en primer lugar la determinación de espectros de diseño de resistencia a partir de la respuesta de S1GL sujetos a una familia de movimientos del terreno que en su conjunto representan la excitación sísmica de diseño. A partir de la respuesta estimada para estos S1GL por medio de análisis paso a paso, pudieran establecerse otros espectros de respuesta, en particular de desplazamiento lateral, de energía plástica disipada, y de daño (los detalles involucrados alrededor de un espectro de daño se discutirán más adelante). Ahora considere el uso del método estático para el diseño de una estructura con periodo fundamental de vibración $T$, capacidad de deformación última caracterizada por una ductilidad $\mu_{u}$, y un coeficiente de amortiguamiento $\xi$. Imagine que pudieran eliminarse todas las incertidumbres involucradas en la estimación de los suministros sísmicos, de tal manera que el valor de las características mecánicas del producto final del proceso de diseño sísmico (la estructura ya diseñada) fuera igual o muy parecido a aquel estimado o supuesto para estas características durante la aplicación del método estático (periodo $T$, capacidad de deformación $\mu_{u}$, coeficiente $\xi$ y un cortante basal igual al producto del peso total de la estructura por el valor de la ordenada correspondiente a $T$ del espectro de diseño de resistencia). Finalmente, considere la posibilidad de llevar a cabo un análisis no lineal paso a paso de la estructura ya diseñada cuando se le sujeta a la misma familia de movimientos del terreno utilizada para establecer los espectros de respuesta, de tal manera que se determinen sus demandas globales de desplazamiento lateral, su demanda de energía plástica disipada, y su nivel de daño estructural. 
En este artículo se plantea, bajo las consideraciones hechas en el párrafo anterior, la comparación de las demandas sísmicas obtenidas en marcos momentorresistentes regulares, sujetos a la acción de una familia dada de movimientos del terreno, con aquellas que acorde a sus características mecánicas, les corresponden según espectros de respuesta establecidos a partir de la misma familia de movimientos. De manera general, puede decirse que si las demandas sísmicas leídas del espectro de respuesta son similares a las demandas sísmicas calculadas directamente en los marcos regulares, entonces es posible plantear que los espectros de respuesta son una herramienta instrumental en la concepción de las estructuras sismorresistentes regulares, y en su diseño sísmico dentro del contexto de un método de análisis estático que contemple el control del daño estructural.

Esta sección estudia la respuesta sísmica de S1GL y de sistemas de varios grados de libertad (SVGL). Para ello, se establece una comparación entre las demandas sísmicas leídas de espectros de respuesta establecidos a partir de S1GL, con las correspondientes demandas estimadas directamente en edificios estructurados con base en marcos momentorresistentes regulares. La resistencia de los S1GL asociados a un espectro de respuesta es tal que su demanda máxima de ductilidad es igual al valor de $\mu$ asociado al espectro. Para los S1GL se presentan espectros de desplazamiento $\left(\delta_{S}\right)$, de energía plástica $\left(E_{P S}\right)$ y de daño $\left(I D_{S}\right)$. Por cada S1GL planteado para obtener los espectros de respuesta, se planteó un edificio regular con el mismo periodo, coeficiente de resistencia lateral (cortante basal máximo normalizado por el peso total del edificio), y coeficiente de amortiguamiento (note que esto es equivalente a plantear que el edificio se ha diseñado conforme al método de análisis estático, y que no ha habido incertidumbre en la estimación de sus características mecánicas). Una vez definidos los edificios, se estimaron mediante análisis no lineales paso a paso sus demandas sísmicas de desplazamiento máximo de azotea $\left(\delta_{V}\right)$, de energía plástica $\left(E_{P V}\right)$ y de daño $\left(I D_{V}\right)$. Tanto para los espectros de respuesta como para los SVGL, se consideraron demandas de energía por unidad de masa. La respuesta de los S1GL se calculó utilizando el método de Newmark con aceleración constante; mientras que aquella de los edificios regulares, utilizando el programa DRAIN 2DX (Prakash et al. 1993).

Para los estudios aquí reportados se consideraron 7 edificios estructurados con base en marcos dúctiles y diseñados conforme al Reglamento de Construcciones para el Distrito Federal. Mientras que la Tabla 2 resume las características mecánicas originales de los edificios, la Figura 11 resume su geometría. Los edificios son simétricos en planta en cuanto a masa, rigidez y resistencia; y no presentan discontinuidades de estas propiedades en elevación, de tal manera que pueden ser considerados regulares. Los edificios originalmente se diseñaron para un factor de comportamiento sísmico de 4 y se consideraron ubicados en la zona del lago del D.F. Para cada edificio se diseñaron dos marcos diferentes, uno interno y uno externo. Cabe destacar que los 7 edificios fueron diseñados por siete alumnos de nivel licenciatura como parte de sus proyectos terminales en la Universidad Autónoma Metropolitana, lo que introduce una sana variabilidad al estudio que aquí se reporta. Una descripción mas detallada de los edificios así como de su procedimiento de diseño puede encontrarse en Terán-Gilmore (1998b).

Originalmente los modelos analíticos de los edificios se plantearon acorde a las consideraciones de modelado discutidas por Teran-Gilmore y Bertero (1993). En resumen, se buscó que los modelos analíticos capturaran de manera adecuada la resistencia, rigidez y capacidad de deformación de los miembros estructurales de los marcos. Para ello, se consideró explícitamente el 
nivel esperado de agrietamiento en vigas y columnas, y el efecto que la losa tiene en la resistencia y capacidad de deformación de las vigas. Debido a las limitaciones de las herramientas de análisis se consideró que: la rigidez de todos los elementos estructurales permanece constante durante la excitación sísmica; las columnas en la planta baja del edificio están empotradas en la base; y los elementos estructurales exhiben un comportamiento elastoplástico. En los modelos analíticos utilizados en este estudio no se consideró endurecimiento por deformación en los elementos estructurales ni efectos $\mathrm{P}-\Delta$.

Para abarcar el rango de resistencias necesario, el coeficiente sísmico de los edificios se igualó al de sus correspondientes S1GL (método estático de análisis), por medio de ajustar de manera proporcional la resistencia de todos sus elementos estructurales. En cuanto al rango de T, el módulo de elasticidad de los modelos analíticos de los edificios se ajustó de manera que, para una primera serie de edificios, el número de pisos $(N)$ fuera igual a $10 T$; mientras que para una segunda serie, igual a $5 T$ (ver Tabla 2). Para los estudios aquí reportados, se utilizaron los acelerogramas sintéticos utilizados en la sección anterior.

El nivel de daño en los S1GL se evaluó utilizando el índice de Park y Ang con un $\beta$ de 0.15 y una $\mu_{\delta u}$ de 4 (ver Ecuación 7). El nivel de daño estructural en los edificios se evaluó con una versión modificada de este índice, que contempla la diferencia entre la capacidad de deformación positiva y negativa de las vigas del edificio conforme a lo siguiente:

$$
I D_{P A}=\max \left(\frac{\theta_{m}^{+}}{\theta_{u}^{+}}, \frac{\theta_{m}^{-}}{\theta_{u}^{-}}\right)+\beta\left(\frac{\theta_{a}^{+}}{\theta_{u}^{+}}+\frac{\theta_{a}^{-}}{\theta_{u}^{-}}\right)
$$

donde la función max denota el mayor de dos valores; $\theta_{u}^{+}$y $\theta_{u}^{-}$son las capacidades últimas de rotación positiva y negativa, respectivamente; $\theta_{m}{ }^{+}$y $\theta_{m}{ }^{-}$son las demandas máximas de rotación plástica positiva y negativa, respectivamente, durante la excitación sísmica; $\theta_{a}^{+}$y $\theta_{a}^{-}$son las demandas acumuladas de rotación plástica positiva y negativa, respectivamente, durante la excitación sísmica; y $\beta$ es una constante que en este caso se consideró igual a 0.15.
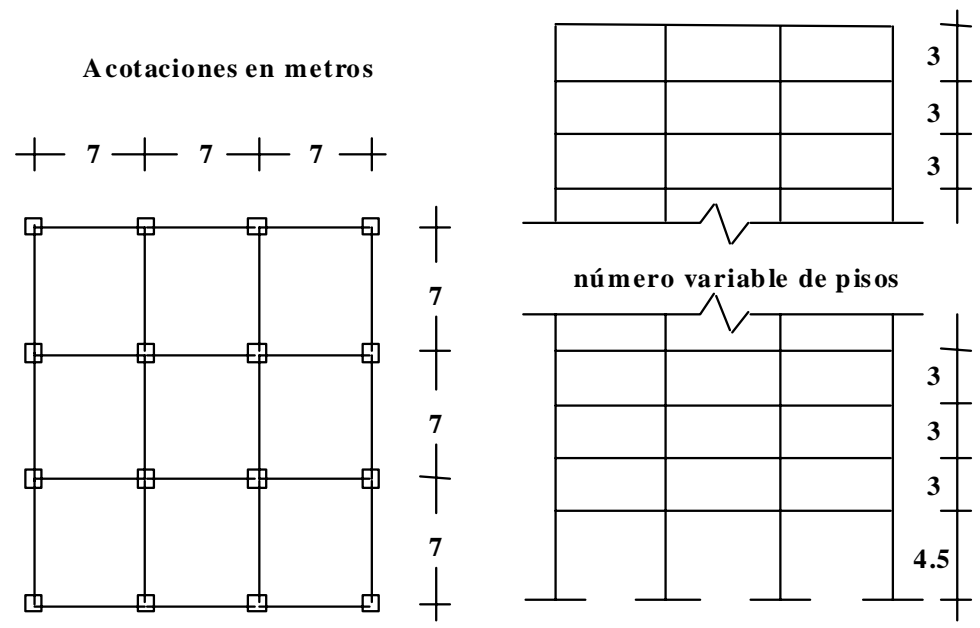

Figura 11 Configuración estructural 
Tabla 2. Características mecánicas de los edificios estudiados

\begin{tabular}{|c|c|c|c|c|c|}
\hline Edificio & $\begin{array}{c}\text { Número } \\
\text { de pisos }\end{array}$ & $\begin{array}{c}\text { Coef. Sísmico } \\
\text { Original }\end{array}$ & $\begin{array}{c}\mathrm{T} \text { (seg) } \\
\text { Original }\end{array}$ & $\begin{array}{c}\mathrm{T} \text { (seg) } \\
\text { Serie 1 }\end{array}$ & $\begin{array}{c}\mathrm{T}(\mathrm{seg}) \\
\text { Serie 2 }\end{array}$ \\
\hline E4 & 4 & .326 & 0.83 & 0.40 & 0.80 \\
\hline E6 & 6 & .221 & 1.17 & 0.60 & 1.20 \\
\hline E8 & 8 & .198 & 1.37 & 0.80 & 1.60 \\
\hline E10 & 10 & .206 & 1.46 & 1.00 & 2.00 \\
\hline E12 & 12 & .227 & 1.48 & 1.20 & 2.40 \\
\hline E16 & 16 & .207 & 1.86 & 1.60 & 3.20 \\
\hline
\end{tabular}

El índice de daño asignado a un edificio dado corresponde al valor máximo entre los valores promedios para el índice de daño de todos los entrepisos. El índice de daño solo se evalúa en las vigas de los edificios. Note que para poder utilizar la Ecuación 16, es necesario definir valores de $\theta_{u}^{+}$y $\theta_{u}^{-}$para un edificio. Para ello se recurrió a llevar a cabo un análisis estático no lineal sujetando cada edificio a un estado de deformaciones monotonicamente crecientes (pushover). Para este análisis se consideró un patrón de fuerzas laterales con la misma distribución que en altura tienen las fuerzas laterales de diseño obtenidas a partir de un análisis modal espectral. A partir de los resultados arrojados por el pushover, se graficó para cada edificio una curva de desplazamiento lateral de azotea contra cortante basal. Como se muestra en la Figura 12, se establece, a partir de una idealización bilineal de esta curva, un desplazamiento de fluencia de azotea $\left(\delta_{y a z}\right)$, el cual hace posible plantear el concepto de ductilidad global $\left(\mu_{g l o b a l}\right)$, definida como el máximo desplazamiento de azotea $\left(\delta_{\operatorname{maxaz}}\right)$ normalizado por $\delta_{\text {yaz }}$. La misma figura ilustra que los valores asignados a $\theta_{u}^{+}$y $\theta_{u}^{-}$corresponden al valor promedio de las rotaciones máximas positivas y negativas en las vigas, respectivamente, cuando $\mu_{\text {global }}$ alcanza un valor de 4.

Para hacer posible una comparación concisa de la respuesta de los SVGL con la de los S1GL, se presenta, como parte de una serie de espectros normalizados, la respuesta de los SVGL, normalizada por su correspondiente ordenada en un espectro de respuesta. En las figuras que se presentan a continuación no se presenta la respuesta normalizada para un sismo particular, sino el promedio de la respuesta normalizada para los 20 acelerogramas sintéticos que conforman un grupo. Para facilitar la interpretación de los espectros normalizados, considere, a manera de ejemplo, que una ordenada de 2 en un espectro de desplazamiento normalizado implica que la demanda de desplazamiento de azotea en los SVGL es el doble de la leída directamente del correspondiente espectro de desplazamientos. Cada uno de los espectros normalizados tiene asignado un valor de $\mu$, que corresponde a la máxima demanda de ductilidad en los S1GL utilizados para obtener los espectros de respuesta (recuerde que los S1GL y SVGL tienen el mismo coeficiente de resistencia lateral). Los resultados se presentan de tal manera que las líneas continuas corresponden a la primera serie de edificios $(\mathrm{T}=\mathrm{N} / 10)$; y las líneas discontinuas a la segunda serie $(T=N / 5)$. 


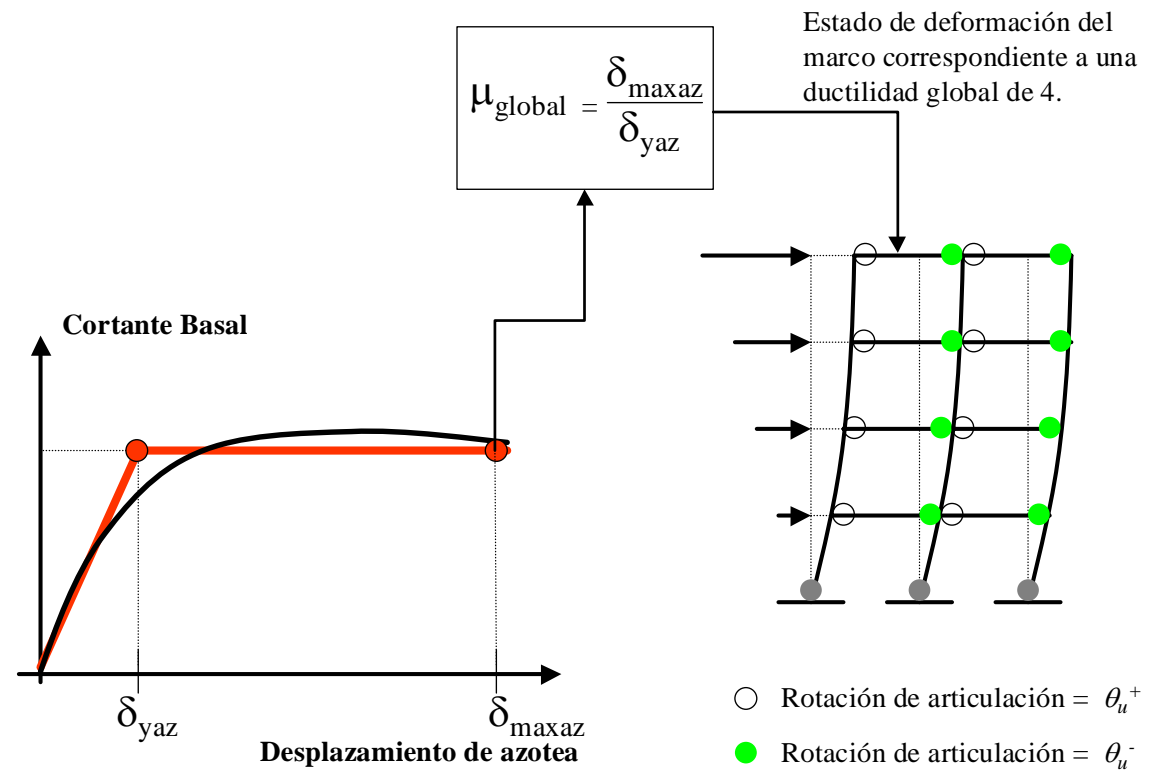

Figura 12 Procedimiento para estimar $\theta_{u}^{+}$y $\theta_{u}$

Las Figuras 13a $(\xi=0.05)$ y $13 \mathrm{~b}(\xi=0.20)$, obtenidas para suelo firme, y las Figuras 13c ( $\xi$ $=0.05)$ y 13d $(\xi=0.20)$, correspondientes a suelo blando, muestran que la relación de desplazamientos tiende a incrementarse ligeramente con un incremento de $T$. De acuerdo a los resultados mostrados, la demanda máxima de desplazamiento lateral de azotea en los edificios regulares $\left(\delta_{V}\right)$ puede obtenerse a partir de la demanda de desplazamiento leída directamente de un espectro de respuesta $\left(\delta_{S}\right)$, si el valor de $\delta_{s}$ se multiplica por $1.4,1.3,1.2$ y 1.1 , para $\mu$ de 1,2 , 3 y 4 , respectivamente. Note que un incremento en el amortiguamiento de los edificios no afecta de manera importante las tendencias discutidas. Lo anterior tiene implicaciones importantes, ya que por un lado sugiere que el desplazamiento máximo en una estructura regular puede estimarse directamente de la información leída directamente de un espectro de desplazamiento; mientras que por otro lado sugiere fuertemente que, para una excitación sísmica de diseño dada, los desplazamientos máximos en los SVGL siguen las mismas tendencias con respecto a los valores de $T, \mu$ y $\xi$, que los desplazamientos máximos de los S1GL utilizados para obtener el espectro de respuesta.

La Figura 14, que se obtuvo a partir de los mismos análisis con que se obtuvo la Figura 13, compara la variabilidad de la demanda máxima de desplazamiento de los edificios con aquella asociada a la respuesta de los S1GL utilizados para establecer el espectro de desplazamiento. Para ello, la Figura 14 presenta la relación entre la desviación estándar de $\delta_{V}\left(\sigma_{\delta v}\right)$ y la desviación estándar de $\delta_{S}\left(\sigma_{\delta \delta}\right)$. Puede notarse que esta relación esta cercana a uno para la mayoría de los casos estudiados, particularmente para las excitaciones de suelo blando. Para suelo firme se presentan casos donde la relación de desviaciones estándar toma valores de 0.6 por un lado y de hasta 1.3 por el otro. El hecho de que la desviación estándar en la respuesta de los SVGL sea muy similar a la asociada a los S1GL implica que las tendencias observadas para la media del desplazamiento en la Figura 13 son similares a las que se observarían para la relación de desplazamientos asociados a cualquier otro nivel de respuesta (media mas una desviación estándar, media mas dos desviaciones estándar, etc.). Esto a su vez tiene implicaciones importantes, ya que sugiere que la incertidumbre 
en la respuesta de los S1GL puede utilizarse directamente para estimar la incertidumbre en la respuesta de SVGL regulares; o en otras palabras, independientemente del nivel de respuesta que se desee estimar (e.g., media, media mas una desviación estándar, etc.), la incertidumbre en la respuesta de los S1GL puede incluirse directamente en el espectro de desplazamientos a partir del cual se desee estimar los valores de $\delta_{V}$.

Las Figuras $15 \mathrm{a}$ ( $\xi=0.05)$ y $15 \mathrm{~b}(\xi=0.20)$, obtenidas para suelo firme, y las Figuras $15 \mathrm{c}$ ( $\xi$ $=0.05)$ y $15 \mathrm{~d}(\xi=0.20)$, correspondientes a suelo blando, muestran que con algunas excepciones, particularmente para suelo blando, la relación de medias de energía plástica por unidad de masa oscila entre valores de 0.8 y 1.0. Lo anterior implica que para una excitación sísmica dada, las demandas de energía plástica en los SVGL regulares exhiben tendencias similares, con respecto a los valores de $T, \mu$ y $\xi$, que las demandas de energía resumidas en un espectro de energía plástica. Como consecuencia, la $E_{P V}$ en un SVGL regular puede estimarse directamente a partir del valor de $E_{P S}$ leído directamente de un espectro de energía plástica. Para el caso de la energía plástica, y considerando la gran incertidumbre asociada a la determinación de las demandas de energía en una estructura sismorresistente, parecería conveniente asignar directamente el valor de $E_{P S}$ a $E_{P V}$. Note que un incremento en el amortiguamiento de los edificios no afecta de manera importante las tendencias observadas, si acaso pudiera decirse que las suaviza un poco, y que dicho incremento se ve reflejado en una ligera reducción en la relación de energías plásticas.

La Figura 16, que se obtuvo a partir de los mismos análisis con que se obtuvo la Figura 15, compara la variabilidad de la demanda de energía plástica en los edificios con aquella asociada a las demandas de energía plástica en los S1GL utilizados para establecer los espectros de respuesta. Para ello, la Figura 16 presenta la relación entre las desviaciones estándares de la energía plástica en los SVGL $\left(\sigma_{E P V}\right)$ y en los S1GL $\left(\sigma_{E P S}\right)$. Note que esta relación oscila, en la mayoría de los casos, entre valores de 0.8 y 1.1, aunque para suelo firme se tienen valores de 0.7 para un rango importante de T. Esto implica que la variabilidad en la respuesta de los SVGL regulares tiene el mismo orden de magnitud que la correspondiente a los S1GL, lo que a su vez implica que en lo que se refiere a la energía plástica, la incertidumbre contemplada directamente en el espectro de energía plástica resulta una medida razonable de aquella correspondiente a la respuesta de los SVGL regulares. Como se discutió antes para el caso del desplazamiento, la incertidumbre en la respuesta de los S1GL puede incluirse directamente en el espectro de energía plástica a partir del cual se desee estimar los valores de $E_{P V}$.

Hasta ahora se ha comentado que las demandas de deformación máxima y acumulada en los SVGL regulares siguen, para una excitación sísmica dada, tendencias muy similares a las observadas para dichas demandas en espectros de respuesta establecidos a partir de S1GL Para estudiar si esta situación también se da en el caso del daño estructural, que depende de las demandas anteriores, las Figuras $17 \mathrm{a}(\xi=0.05)$ y $17 \mathrm{~b}(\xi=0.20)$, obtenidas para suelo firme, y las Figuras $17 \mathrm{c}(\xi=0.05)$ y $17 \mathrm{~d}(\xi=0.20)$ correspondientes a suelo blando, presentan la relación de medias del índice de daño de Park y Ang. Puede observarse que con unas cuantas excepciones, particularmente en el caso de suelo blando, dicha relación oscila entre valores de 0.7 y 0.9 para $\xi$ de 0.05 . Esto tiene implicaciones muy importantes, ya que sugiere que el daño en estructuras regulares puede estimarse a partir de información leída directamente de espectros de respuesta. Note que un incremento en el amortiguamiento de los edificios tiende a aumentar la dispersión de los valores de la relación de medias del índice de daño de Park y Ang. 


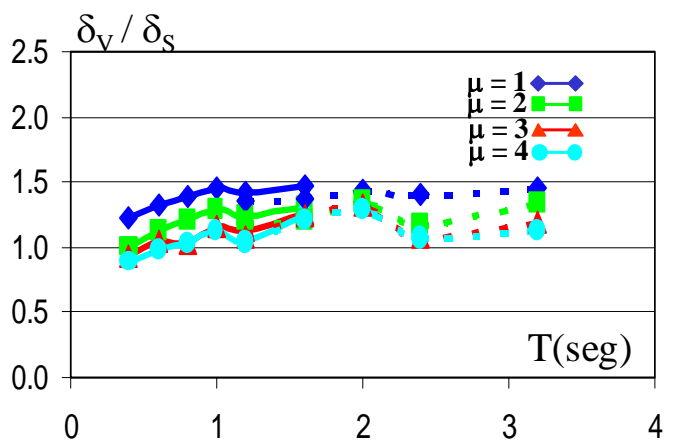

a) Suelo firme, $\xi=0.05$

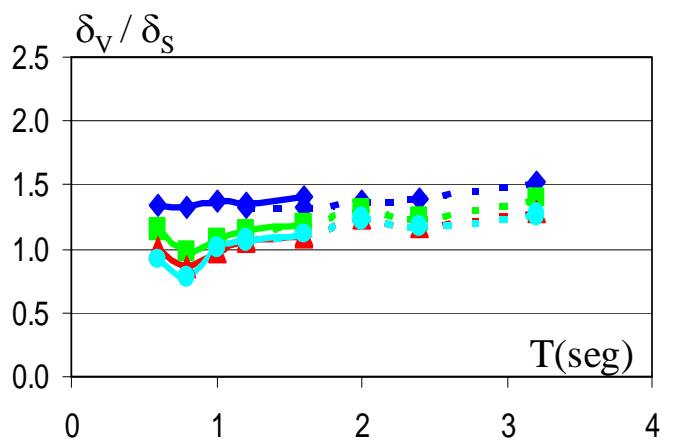

c) Suelo blando, $\xi=0.05$

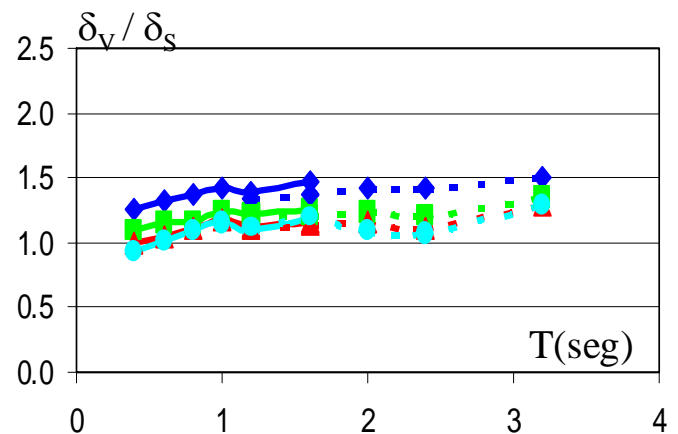

b) Suelo firme, $\xi=0.20$

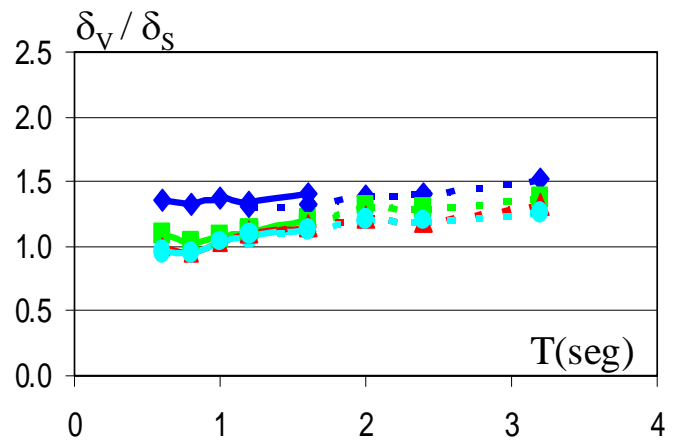

d) Suelo blando, $\xi=0.20$

Figura 13 Relación de medias de desplazamiento lateral
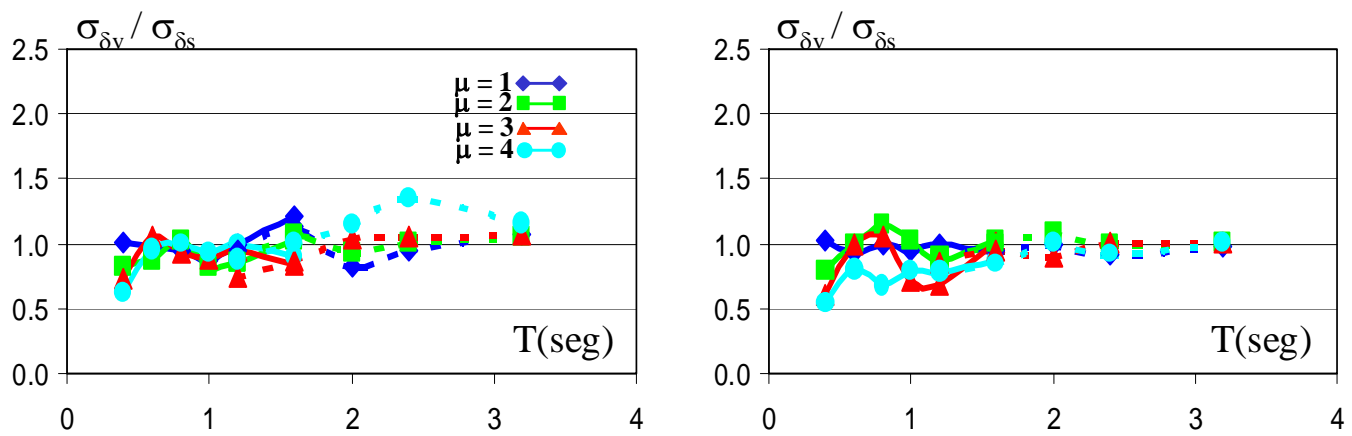

a) Suelo firme, $\xi=0.05$

b) Suelo firme, $\xi=0.20$
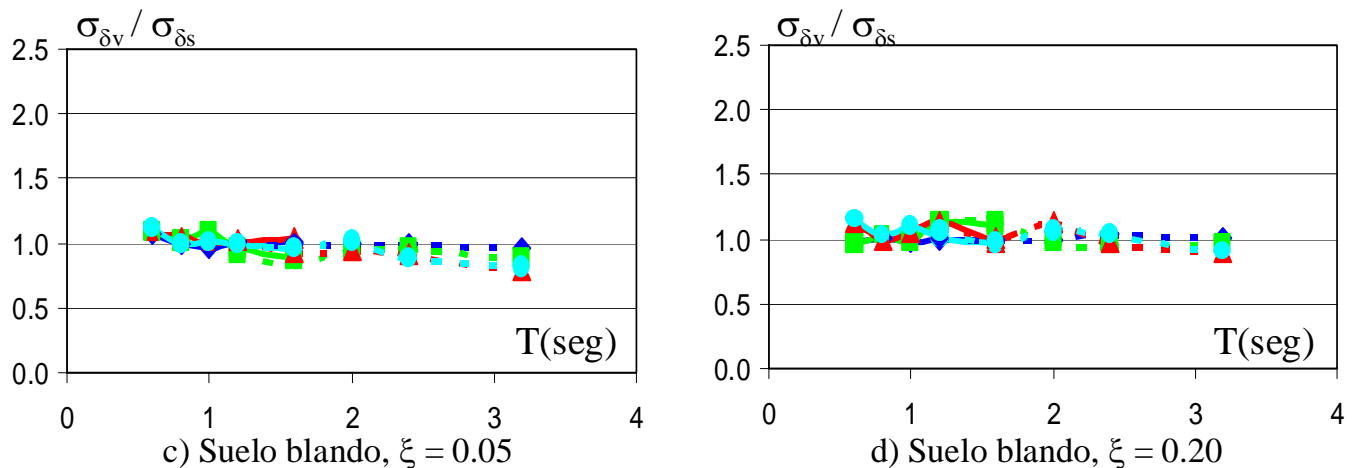

Figura 14 Relación de desviaciones estándar de desplazamiento lateral. 
La Figura 18, que se obtuvo a partir de los mismos análisis con que se obtuvo la Figura 17, compara la variabilidad del valor del índice de Park y Ang en los edificios con aquella asociada al mismo índice en los S1GL utilizados para establecer los espectros de respuesta. Para ello, la Figura 18 presenta la relación entre las desviaciones estándares de los valores del índice en los SVGL $\left(\sigma_{I D V}\right)$ y en los S1GL $\left(\sigma_{I D S}\right)$. Note que la relación de desviaciones estándares del daño suele tener valores significativamente mayores que uno, y que alcanza valores cercanos a 4. Además, el valor de la relación de desviaciones estándares exhibe incrementos importantes con un decremento en el valor de $\mu$. A diferencia de lo que ocurre para las demandas máximas y acumuladas de deformación, la variabilidad en el valor del índice de daño en los SVGL regulares es mucho mayor que aquella asociada a la respuesta de los S1GL. Lo anterior implica que las relaciones mostradas en la Figura 17 no pueden aplicarse directamente para asociar el daño de los SVGL y S1GL para otros niveles de respuesta diferentes a la media (e.g., media mas una desviación estándar, media mas dos desviaciones estándar, etc.). Para ilustrar lo anterior, la Figura 19 muestra las relaciones existentes entre la media mas una desviación estándar y la media más dos desviaciones estándar de los valores del índice de daño en los SVGL y los S1GL. Note que conforme se considera un nivel de respuesta que involucra mas desviaciones estándar, el valor de la relación de índices de daño tiende a aumentar. Este aumento se da de tal manera que para los niveles de respuesta mostrados en la Figura 19, el valor de dicha relación oscila alrededor de valores muy cercanos a uno. Esto implica que para las familias de acelerogramas utilizadas y para niveles de daño asociados a un nivel de respuesta que considere la media mas una o dos desviaciones estándar, la información leída directamente de un espectro de respuesta provee una excelente base para estimar, de una manera confiable, el nivel de daño en estructuras regulares.

Antes de concluir esta sección, es importante destacar dos hechos. Primero, para el caso de las excitaciones de suelo blando y $T$ ligeramente menor a $0.5 T_{g}$, la relación de medias de índice de daño es considerablemente menor que uno, lo que implicaría que derivar información de daño en este rango de $T$ a partir de espectros de respuesta resulta muy conservador. Esta tendencia también se ha observado en el estudio de la respuesta de SVGL simples, formados por masas conectadas entre sí por resortes que modelan las características mecánicas de entrepiso (Terán-Gilmore 1999). Note que conforme a lo planteado al principio de esta sección, el índice de daño asignado a un edificio dado corresponde al valor máximo entre los valores promedios para el índice de daño de todos los entrepisos, de tal manera que los resultados resumidos en las Figuras 17 y 19 contemplan el hecho de que unos pisos dentro de una estructura se dañan mas que otros. Segundo, las observaciones hechas en esta sección son válidas para los acelerogramas sintéticos y los edificios regulares considerados en el estudio aquí reportado. Aunque se ha hecho una caracterización razonable, en cuanto a forma espectral y contenido de frecuencias, de las excitaciones sísmicas generadas en suelos firmes y blandos por medio de acelerogramas sintéticos, es importante hacer notar que la variabilidad en las características dinámicas de acelerogramas reales es mayor que aquella asociada a dichas características en acelerogramas sintéticos. A partir de esto, se aclara que algunas de las conclusiones presentadas aquí pudieran variar ligeramente según la muestra de acelerogramas utilizada. Al respecto, Terán-Gilmore (1999) ha observado, a través de la comparación de resultados obtenidos en acelerogramas reales y sintéticos, que las tendencias aquí descritas también se observan para acelerogramas reales. Finalmente, también conviene notar que conforme una estructura exhiba mayor irregularidad estructural, algunas de las observaciones hechas en este artículo podrían no ser aplicables directamente a ella. 


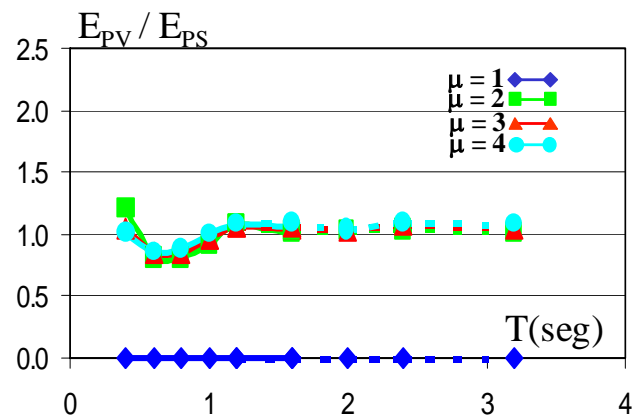

a) Suelo firme, $\xi=0.05$

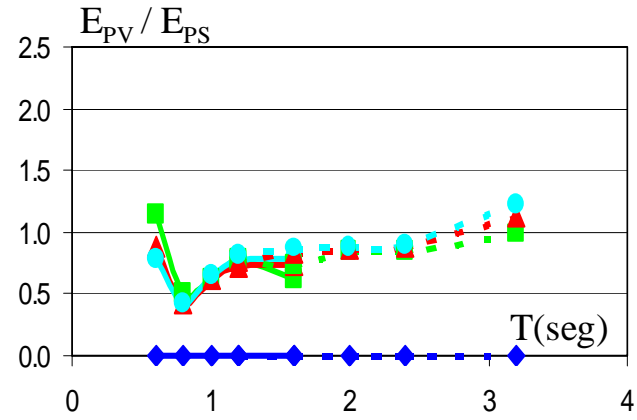

c) Suelo blando, $\xi=0.05$

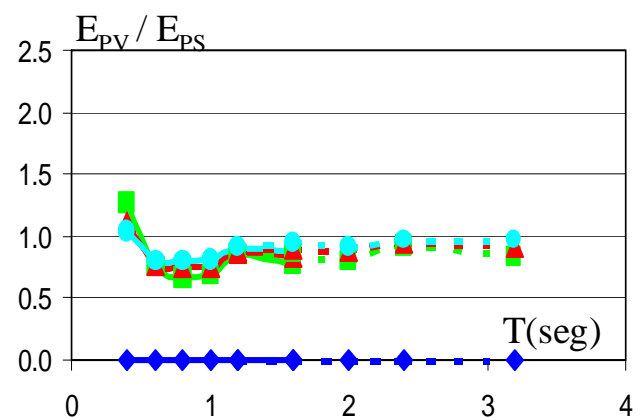

b) Suelo firme, $\xi=0.20$

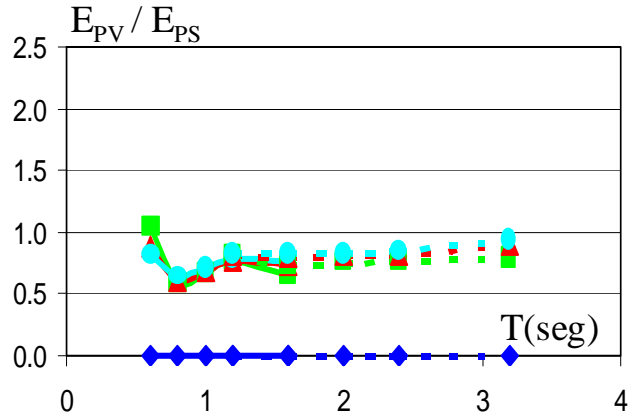

d) Suelo blando, $\xi=0.20$

Figura 15 Relación de medias de energía plástica

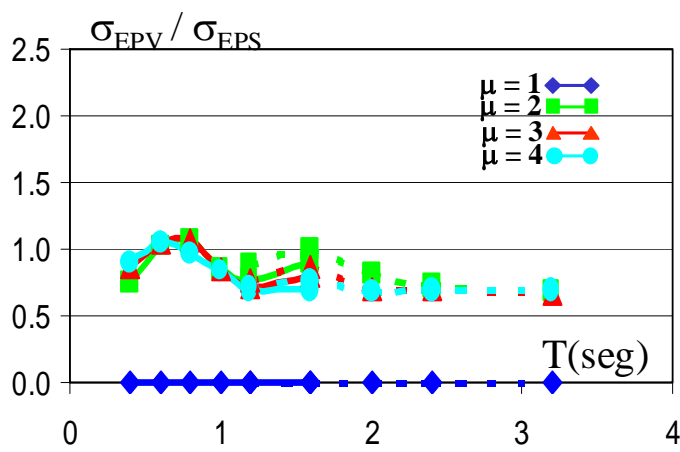

a) Suelo firme, $\xi=0.05$

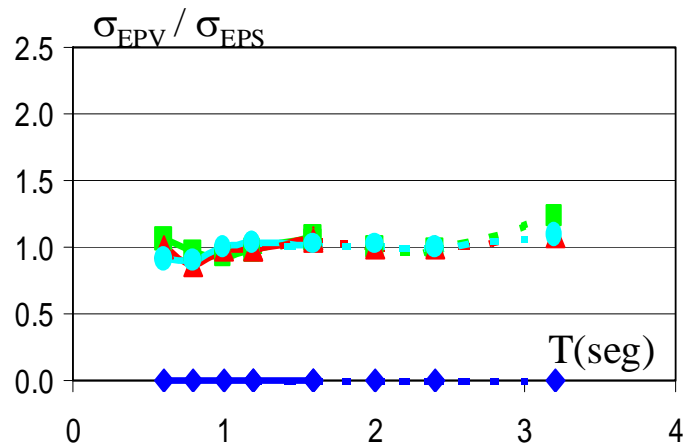

c) Suelo blando, $\xi=0.05$

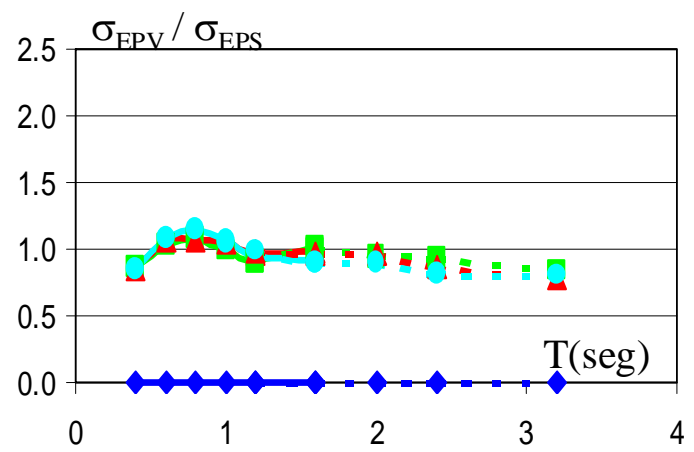

b) Suelo firme, $\xi=0.20$

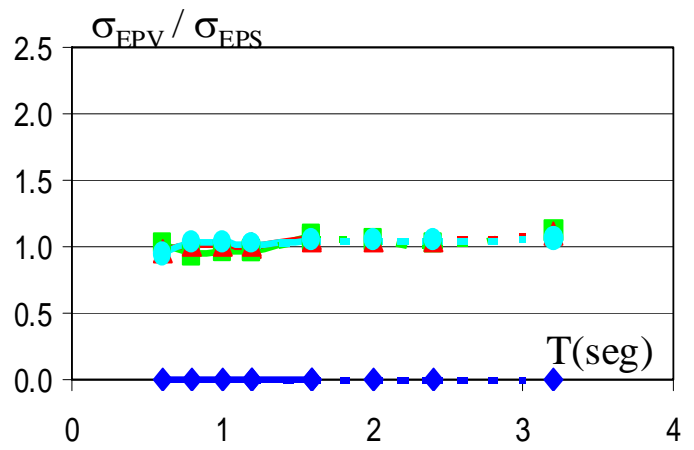

d) Suelo blando, $\xi=0.20$

Figura 16 Relación de desviaciones estándar de energía plástica 

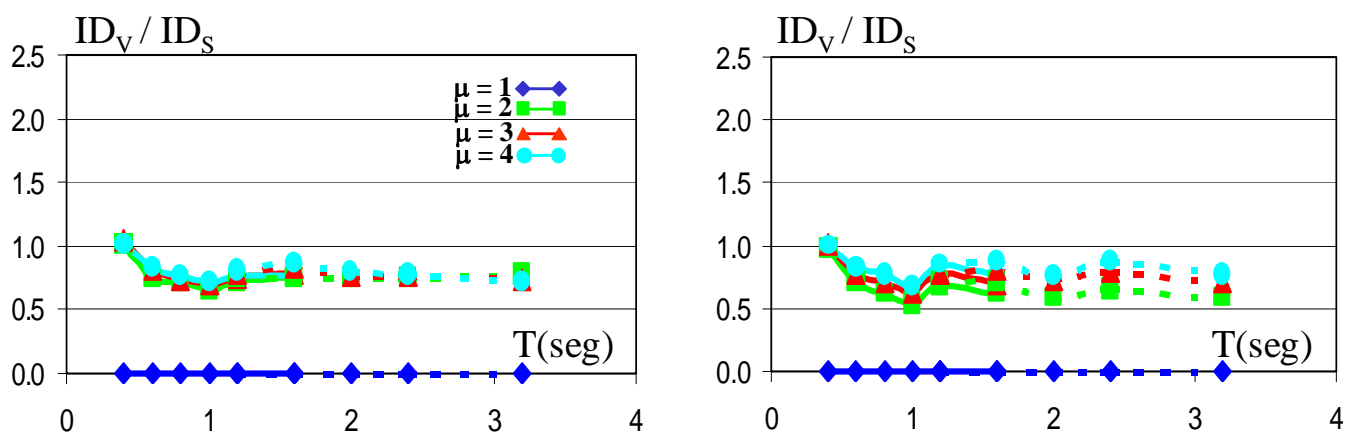

a) Suelo firme, $\xi=0.05$

b) Suelo firme, $\xi=0.20$

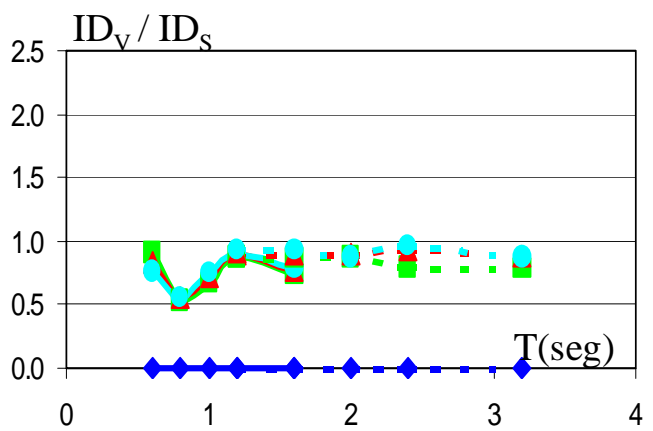

c) Suelo blando, $\xi=0.05$

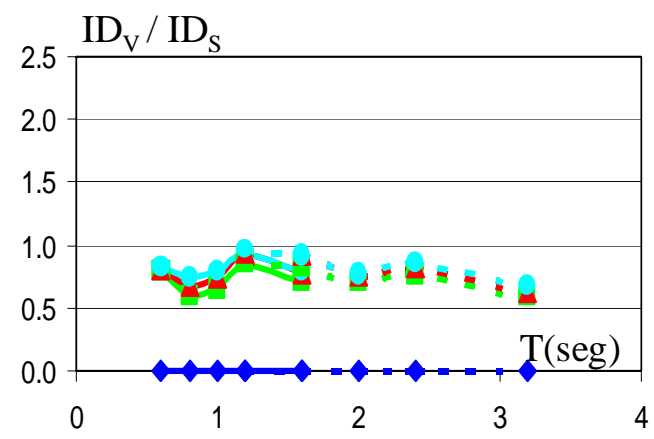

d) Suelo blando, $\xi=0.20$

Figura 17 Relación de medias de índice de daño

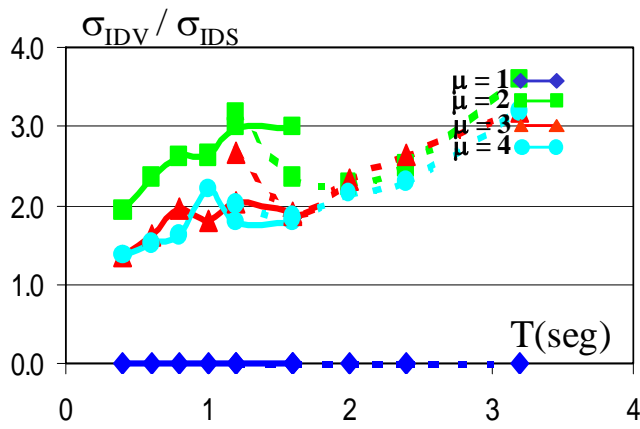

a) Suelo firme, $\xi=0.05$

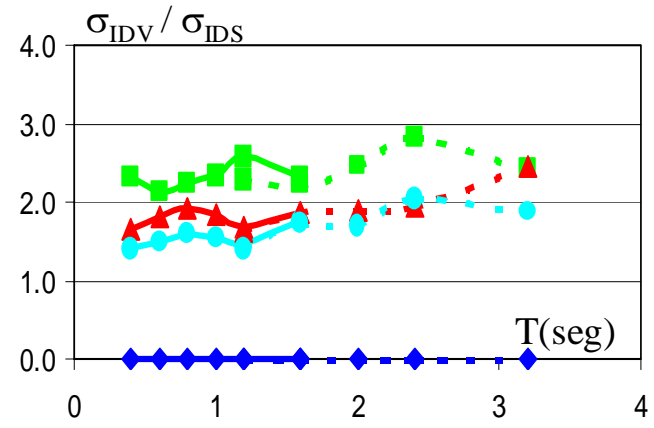

b) Suelo firme, $\xi=0.20$

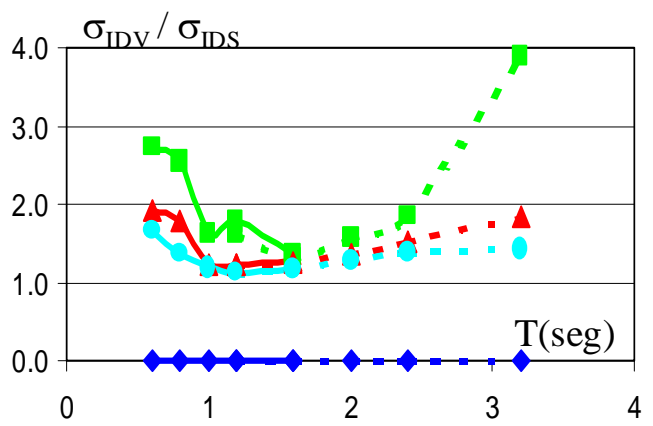

c) Suelo blando, $\xi=0.05$

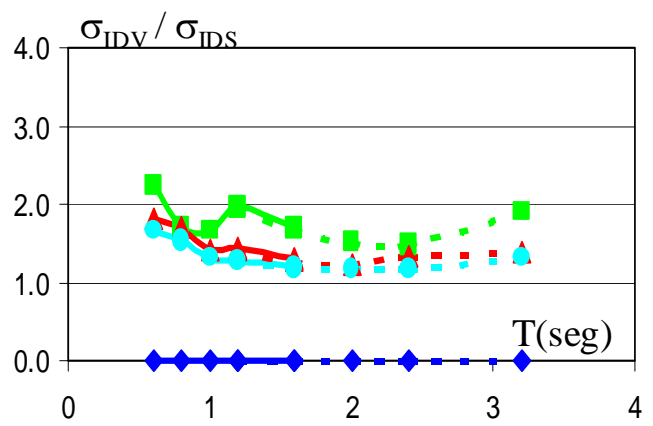

d) Suelo blando, $\xi=0.20$

Figura 18 Relación de desviaciones estándares de índice de daño 


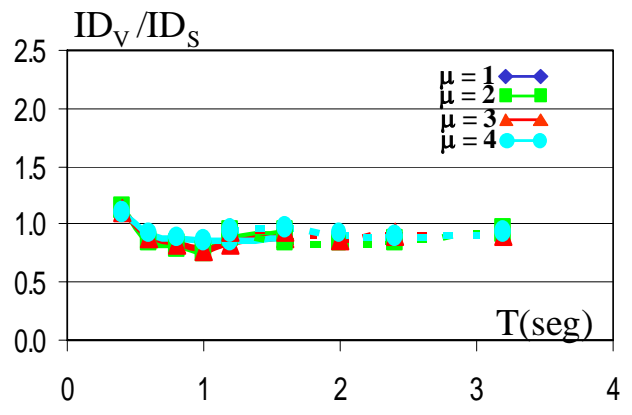

a) Suelo firme, Media $+\sigma$

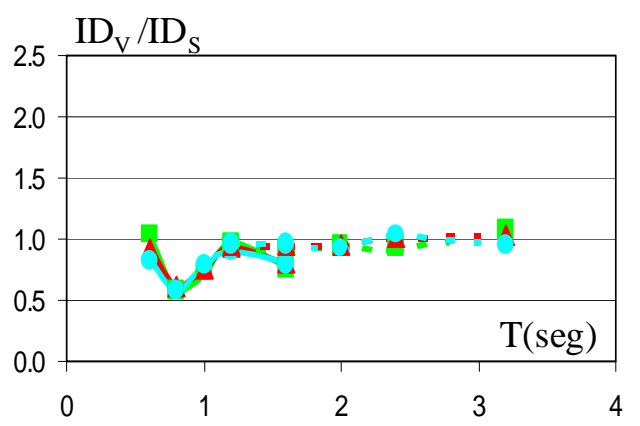

c) Suelo blando, Media $+\sigma$

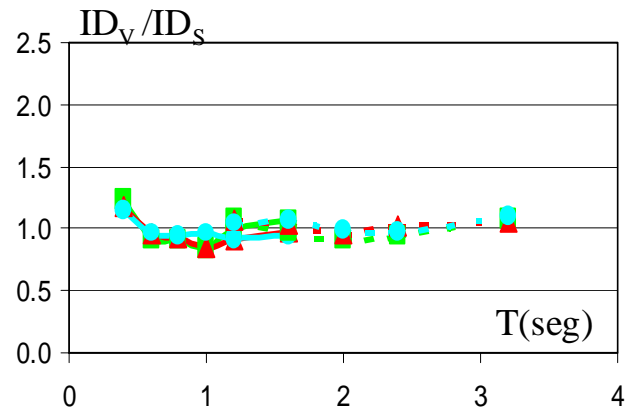

b) Suelo firme, Media $+2 \sigma$

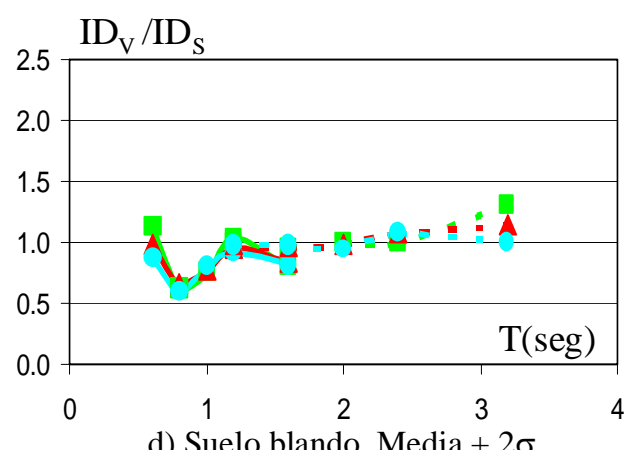

d) Suelo blando, Media $+2 \sigma$

Figura 19 Relación de otros niveles de índice de daño, $\xi=0.05$

\section{OBSERVACIONES Y CONCLUSIONES}

Aunque existen diferentes enfoques para plantear índices de daño estructural, la mayoría de ellos considera alguna medida de las demandas de deformación en la estructura sismorresistente. Algunos de estos índices consideran la acumulación de deformación plástica. Dentro de este último grupo, pueden identificarse dos enfoques, que consisten en considerar o ignorar la forma en que la estructura disipa la energía plástica durante la excitación sísmica.

Un diseño sísmico no contempla el diseño de la estructura contra una excitación sísmica particular; sino que esta debe diseñarse para que, con cierto grado de confiabilidad, resista toda una familia de excitaciones sísmicas que en su conjunto representan la excitación sísmica de diseño. Dentro de este contexto, los resultados presentados en este artículo sugieren que la energía plástica tiende a disiparse de una manera bien definida para una familia dada de sismos (el número de ciclos crece notoriamente con una disminución de su ductilidad). La disipación de energía no se da en secuencias “extremas” de acumulación de demandas plásticas de deformación, sino que sigue tendencias muy similares a las que exhiben las curvas típicas de capacidades de disipación de energía plástica (como la mostrada en la Figura 3b). Con base en esto, puede afirmarse que en la mayoría de los casos, no es necesario conocer el número y magnitud de los ciclos de comportamiento plástico para estimar el nivel de daño estructural en las estructuras sismorresistentes. Esto a su vez sugiere que la energía plástica que disipa la estructura cuando se le sujeta a la familia de excitaciones sísmicas de diseño resulta información suficiente para evaluar el efecto que la fatiga de bajo ciclaje tiene en su desempeño estructural. Dentro de este contexto, el uso del índice de Park y Ang, que considera el efecto de las demandas 
acumuladas de deformación plástica a través de la energía plástica disipada, puede utilizarse para plantear el control de daño estructural durante un diseño sísmico por desempeño. Ejemplo de lo anterior son los planteamientos hechos por Bertero y Bertero (1992), y por Bertero, Bertero y Teran-Gilmore (1996).

Índices de daño como el de Park y Ang pueden utilizarse de manera confiable para estimar el nivel de daño estructural en estructuras desplantadas en suelo firme. Para el caso de excitaciones generadas en suelo blando, el uso de este tipo de índices resulta en una subestimación del nivel de daño para estructuras cuyo periodo fundamental de vibración este cercano al periodo característico de la excitación; esta subestimación tiende a desaparecer conforme el periodo de la estructura se aleja del periodo predominante de la excitación. Parecería que la única consideración que debe hacerse para utilizar el índice de Park y Ang para estimar daño en estructuras desplantadas en suelo blando consiste en incrementar las demandas de resistencia obtenidas a partir de dicho índice (alrededor de $20 \%$ en caso de que el nivel de daño sea colapso incipiente) para un periodo igual al periodo dominante de la excitación, y reducir paulatinamente este incremento hasta un valor de cero para estructuras cuyo periodo se aleje decisivamente de aquel de la excitación.

Para una excitación sísmica dada, las demandas sísmicas de deformación, tanto máxima como acumulada, en SVGL regulares siguen tendencias muy similares a las observadas en espectros de respuesta establecidos a partir de S1GL. De hecho, las demandas de deformación en SVGL regulares y aquellas resumidas en espectros de respuesta exhiben dependencias similares con respecto a los valores de $T, \mu$ y $\xi$. A partir de esto, es posible concluir que el daño estructural en estructuras regulares puede estimarse directamente a partir de la información derivada de espectros de respuesta. Al respecto, es posible anticipar que el prediseño de estructuras regulares dentro del marco de un diseño sísmico basado en control de daño, puede llevarse a cabo mediante el uso de espectros de respuesta de desplazamiento, energía plástica y otros, conforme a lo discutido por Teran-Gilmore (1998a).

La variabilidad de la respuesta dinámica de las estructuras sismorresistentes ante una familia dada de excitaciones sísmicas puede ser incorporada directamente a los espectros de diseño a partir de la variabilidad en la respuesta de S1GL. Al respecto, basta tener en cuenta la incertidumbre en la respuesta de los S1GL durante la definición de los espectros de diseño, para obtener una representación confiable de la excitación sísmica de diseño para el diseño sísmico de estructuras regulares.

Finalmente, conviene mencionar que el alcance de las observaciones y conclusiones presentadas aquí está limitado a edificios regulares. Por tanto, es necesario ampliar las investigaciones hechas aquí para identificar bajo que condiciones es necesario llevar a cabo un análisis dinámico completo de la estructura para tomar en cuenta el efecto de la fatiga de bajo ciclaje.

\section{AGRADECIMIENTOS}

Parte de los resultados presentados en este artículo fueron obtenidos por Esmeralda Ávila como parte de su tesis de maestría. Se agradece su entusiasmo y dedicación. 


\section{REFERENCIAS}

Ávila, E. y Terán-Gilmore, A. (2000). "Efecto de la historia de disipación de energía en el desempeño estructural”, Memorias del XII Congreso Nacional de Ingeniería Estructural (publicadas en $\mathrm{CD}$ ).

Bertero, R.D y Bertero, V.V. (1992). “Tall reinforced concrete buildings: conceptual earthquakeresistant design methodology”, Reporte UCB/EERC-92/16, Department of Civil Engineering, Universidad de California en Berkeley.

Bertero, R.D. y Bertero, V.V. (2000). "Application of a comprehensive approach for the performance-based earthquake-reistant design of buildings", Memorias del Doceavo Congreso Mundial de Ingeniería Sísmica (publicadas en CD).

Bertero, R. D., Bertero, V.V. y Teran-Gilmore, A. (1996). "Performance-based earthquakeresistant design based on comprehensive design philosophy and energy conceps", Memorias del Onceavo Congreso Mundial de Ingeniería Sísmica (publicadas en CD).

Chen, Y. y Gong, S. (1986). "Double control damage index of structural ductility and dissipated energy during earthquake”, Journal of Building Structure, 35-48.

Chai, Y.H., Romstad, K.M. y Bird, S.M. (1992). "Energy-based linear damage model for highintensity seismic loading”, Journal of Structural Engineering, ASCE, Vol. 121, 857-864.

Cosenza, E., Manfredi, G. y Ramasco, R. (1990). “An evaluation of the use of damage funtionals in earthquake-resistant design”, Memorias del Onceavo Congreso Europeo de Ingeniería Sísmica, Vol. 9, 303-312.

Fajfar, P. y Fischinger, M. (1992). "An approximate method for seismic damage analysis of buildings”, Memorias del Décimo Congreso Mundial de Ingeniería Sísmica, Vol. 7, 3921-3926.

Fajfar, P. y Krawinkler, H. - Editores - (1997). "Seismic Design Methodologies for the Next Generation of Codes", Memorias del Sexto Congreso SECED, Vol. 1, 459-466.

Hodder, S. B. (1983). "Computer processing of New Zealand strong-motion accelerograms", Tercer Congreso Regional de Ingeniería Sísmica del Pacifico Sur, Vol. 1, 36-53.

Lai, S. P. (1982). "Statistical characterization of strong ground motions using power spectral density function”, Bulletin of the Seismological Society of America, Vol. 72, No 1, 259-274.

Nassar, A.A. y Krawinkler, H. (1991). "Seismic demands for SDOF and MDOF systems", Reporte No. 95, The John A. Blume Earthquake Engineering Center, Department of Civil Engineering, Stanford.

Park, Y. J. y Ang, A. H. (1985). "Mecanistic seismic damage model for reinforced concrete", Journal of Structural Engineering, ASCE, Vol 111, N ST4, 740-757. 
Prakash, V., Powell, G.H. y Campbell, S. (1993). "DRAIN-2DX Base program description and user guide", Reporte UCB/SEMM-93/17, Department of Civil Engineering, Universidad de California en Berkeley.

Qi, X. y Moehle, J.P. (1991). "Displacement design approach for reinforced concrete structures subjected to earthquakes”, Reporte UCB/EERC-91/02, Department of Civil Engineering, Universidad de California en Berkeley.

Tajimi, H. (1960). “A statistical method for determining the maximum response of a building structure during an earthquake”, Memorias del Segundo Congreso Mundial de Ingeniería Sísmica, Vol. II, 781-797.

Teran-Gilmore, A. y Bertero, V.V. (1993). "Seismic performance of a 30-story building located in soft soil and designed according to UBC 1991”, Reporte UCB/EERC-93/04, Department of Civil Engineering, Universidad de California en Berkeley.

Teran-Gilmore, A. (1996). "Performance-based earthquake-resistant desing of framed buildings using energy concepts”, Tesis de Doctorado, Department of Civil Engineering, Universidad de California en Berkeley.

Teran-Gilmore, A., Juárez, H. y Frausto, M. (1998). "Efectos de la asimetría en fluencia en el comportamiento sísmico de sistemas estructurales”, Revista de Ingeniería Sísmica № 58, 41-63.

Teran-Gilmore, A. (1998a). “A parametric approach to performance-based numerical seismic design”, Earthquake Spectra, Vol. 14, N 3, 501-520.

Teran-Gilmore, A. (1998b). "Características dínamicas y desempeño sísmico de marcos dúctiles de concreto reforzado", Memorias del XI Congreso Nacional de Ingeniería Estructural, Vol. 1, 564573.

Teran-Gilmore, A. (1999). "Predicción de la respuesta sísmica de marcos regulares", Memorias del XII Congreso Nacional de Ingeniería Sísmica, Vol. II, 1146-1155.

Trifunac, M.D. y Bray, A.G. (1975). “A study on the duration of strong earthquake ground motion”, Bulletin of the Seismological Society of America, Vol. 65, N 3, 581-626.

Tung, A., Wang, J. N., Kiremidjian, A. S. y Kavazanjian, E. (1992). "Statistical parameters of AM and PSD fuctions for the generation of site-specific strong ground motions", Memorias del Décimo Congreso Mundial de Ingeniería Sísmica, Vol. 2, 867-872.

Williams, M.S. y Sexsmith, R.G. (1995). "Seismic damage indices for concrete structures”, Earthquake Spectra, Vol. 11, N² 2, 319-349. 